\title{
28 Research Square \\ The Mechanism of Flower Opening and Closing Times Revealed By Transcriptome Profiling
}

\author{
Rong Liu \\ Beijing Forestry University \\ Chunjing Guan \\ Beijing Forestry University \\ Zhuping Fan \\ Beijing Forestry University \\ Liya Ding \\ Beijing Forestry University \\ Yike Gao ( $\nabla$ gaoyk@bjfu.edu.cn ) \\ Beijing Forestry University \\ Qixiang Zhang \\ Beijing Forestry University
}

\section{Research Article}

Keywords: Circadian clock, Flower opening time, Flower closing time, Hormone, PCD, Transcriptome

Posted Date: November 16th, 2021

DOI: https://doi.org/10.21203/rs.3.rs-1043621/v1

License: (1) This work is licensed under a Creative Commons Attribution 4.0 International License. Read Full License 


\section{Abstract}

Background: Circadian clock precisely control flowers open and close at specific times of day responsive to the environment, providing advantages in reproduction and adaption. Flowers of Iris dichotoma and I. domestica are ephemeral and show single opening and closing which present circadian rhythms. To reveal the key clock-related genes and mechanisms regulating flower opening and closing times, transcriptomes of two F2 hybrid plants with significantly divergent flower opening and closing times were sequenced at different flowering stages. Genes with different temporal expression patterns between the two individuals were screened.

Results: PIF4-like (PHYTOCHROME-INTERACTING FACTOR4-like) whose homologous gene in Arabidopsis integrates external signals (light and temperature) and intrinsic signals from the clock would negatively regulate flower opening and positively regulate flower closing. Downstream the clock, genes related to auxin synthesis (YUC10 and YUC2-like), auxin efflux transport (auxin efflux carrier component 1,7$)$ and signaling (SAUR64-like and auxin-induced protein 22D) were specifically up-regulated in flower opening process (from flower buds to flower starting opening), suggesting that auxin is necessary for flower opening. Genes involved in osmoregulation, inward water transport and cell wall loosening were also specifically up-regulated in flower opening process, causing rapid cell expansion during flower opening. The start of flower closure is associated with the increased activities of cell wall degradation, protein degradation, sucrose synthesis, fatty acid degradation, phospholipid degradation and remobilization of mineral ions.

Conclusions: This research illuminates the key clock-related regulator of flower opening and closing times, and improves the understanding in regulatory networks of flower opening and closing.

\section{Background}

Flower opening and closing are closely associated with successful sexual propagation because they determine whether pollination can occur $[1,2]$. Temporal partitioning of flowering times within a day caused by markedly different flower opening and closing times among species has a key role in the reproductive isolation among sympatric species [3, 4]. Therefore, knowledge about the regulatory mechanism of divergent flower opening and closing times among species is important to understand the evolution of species. Furthermore, understanding the regulatory mechanism facilitates to control flower opening and closing times accurately at molecular levels, which has considerable commercial potential. For example, flower opening earlier helps rice escape high temperature stress $\left(35-40^{\circ} \mathrm{C}\right)$ and thus improves the availability of pollination, which can increase the yield of rice [5, 6]. Delaying flower closing (senescing) time can extend the floral longevity of cut flowers. Despite the apparent significance, few studies focus on the regulatory mechanism of differential flower opening and closing times among species or individuals.

Flower opening and closing of certain plants constantly occur at particular times of the day, showing circadian rhythm. These rhythms can keep stable under constant light or dark conditions, demonstrating the involvement of circadian clocks [1, 7-9]. The regulation of circadian clock in flower opening time has been verified at the molecular level [10]. Furthermore, significant transcript changes of abundance of clock-related genes during flower opening and closing also reveal the regulation of circadian clock in flower opening and closing $[11,12]$.The circadian clock system perceives and responses to external signals. Then these signals are integrated by the central oscillator and are transferred to output networks for the regulation of numerous downstream rhythmic physiology and behaviors [13,14], such as rhythmic flower opening and closing. The molecular mechanism of core oscillator and light input pathways have been well understood in model plant Arabidopsis thaliana [15-17]. However, it is not known how clock regulates flower opening and closing times in response to light signals.

Compared with the poor understanding of upstream regulatory mechanisms of flower opening and closing, the downstream physiological mechanisms of flower opening and closing have been relatively well understood $[1,2]$. Flower opening relies on petal movements which are generally induced by cell expansion [1]. Cell expansion is regulated by hormones, water transport, osmoregulation, sugar metabolism and cell wall extensibility $[1,2,18]$. Flower closure or flower senescence of ephemeral flowers is considered to be a type of programmed cell death (PCD) [19]. Flower senescence is generally accompanied with turgor loss, cell wall degradation, protein degradation, fatty acid degradation, phospholipid degradation and many other physiological changes $[19,20]$. Moreover, a number of downstream genes related to flower opening and closing behaviors have been detected through analyzing the changes of gene expression during opening and (or) closing processes in a species [11, 12, 21-23]. However, the key clock-related genes controlling flower opening and closing times have not been reported. This may be resulted from lacking lateral comparison of 
transcriptome changes in flower opening and closing processes between different plants which have the same genetic background but markedly divergent flower opening and closing times.

I. dichotoma and I. domestica are two wild Iris species which have stable flower opening and closing rhythms. These rhythms kept robust even in the constant dark condition (unpublished data), suggesting the involvement of circadian clock. Flowers of the two species are ephemeral and show single opening and closure [24], which means the closure is caused by senescence. Flower opening and closing times differ largely between I. dichotoma (starting opening at 16:00 and starting closing at 20:30) and I. domestica (starting opening at 7:00 and starting closing at 17:00), which becomes a key factor driving reproductive isolation between the two species [4]. To obtain individuals with the same genetic background but different flower opening and closing times for transcriptome sequencing, the F1 (I. dichotomax I. domestica) and F2 hybrid populations between the two species were established. Flower opening and closing times have low variability in the F1 hybrids but high variability in the F2 hybrids [4]. Due to the stability and high variability of the F2 hybrids in flower opening and closing times as well as the same genetic background, the F2 hybrids of $I$. dichotoma and $I$. domestica provide an extraordinary opportunity to investigate the key genes controlling flower opening and closing times and the regulatory mechanisms of flower opening and closing. Furthermore, the high visibility of flower opening and closing rhythms, and short growth cycles (it takes only a year from sowing to flowering for I. dichotoma and I. domestica) provides great convenience for the investigation of circadian rhythm.

The aims of this study are to identify the upstream clock-related key genes which regulate flower opening and closing times, and clarify the regulatory pathways of flower opening and closing. We also have discussed the reason causing differential flower opening and closing times between different individuals. For these purpose, two individuals with significantly different flower opening and closing times were selected from the F2 hybrids of I. dichotoma and I. domestica, which were named as MA (morning-opening and afternoon-closing plant) and $\mathrm{AE}$ (afternoon-opening and evening-closing plant). RNA-sequencing was conducted in MA and $\mathrm{AE}$ at four time points of the day (T1, T2, T3 and T4) which are flower opening and closing times of the two F2 plants (Table 1, Fig. 1). Then we screened genes with different expression profiles between the two individuals on the same time course but different flowering course, because these genes may be closely related to flower opening and closing.

\section{Results}

Eight libraries were sequenced using the Illumina Hiseq 2000 platform (Table 1, Fig. 1). After filtering the raw data, a total of $535,683,215$ clean reads were obtained. Of these reads, $130,705,691$ reads with an average GC content of 50.08\% were from MA-T1 and MA-T2, 126,431,320 reads with an average GC content of 50.03\% were from AE-T1 and AE-T2, 138,068,167 reads with an average GC content of $50.64 \%$ were from MA-T3 and MA-T4, and 140,478,037 reads with an average GC content of $49.61 \%$ were from AE-T3 and AE-T4 (Table S1). Q30 percentage (percentage of bases with sequencing error rate lower than 1\%o) were $93.88 \%$ for MA-T1 and MA-T2, 93.94\% for AE-T1 and AE-T2, 95.54\% for MA-T3 and MA-T4, 92.22\% for AE-T3 and AE-T4. These data showed that the RNASeq quality was applicable for further analysis.

After the assembly of the clean reads, a total of 57949 unigenes were generated from T1 and T2, which had an average length of 1276 bp and N50 length of 2023 bp (Table S2). A total of 53,699 unigenes were obtained from T3 and T4, which had an average length of $1419 \mathrm{bp}$ and N50 length of $2329 \mathrm{bp}$ (Table S2). In both unigene libraries, unigenes between 300bp and 500bp had the largest percentage (Fig. S1 and Fig. S2). These data indicated the high quality of the assembled unigenes.

A total of 33812 unigenes (58.35\%) from T1 and T2, 32469 unigenes $(60.46 \%)$ from T3 and T4 were annotated to Nr, Swissprot, GO, KEGG, COG and Pfam database. Among the annotated unigenes from T1 and T2, 33243 unigenes (98.32\%) were annotated to Nr, 23920 (70.74\%) to Swissprot, 19239 (56.90\%) to GO, 13752 (40.67\%) to KEGG, 10885 (32.19\%) to COG and 23397 (69.20\%) to Pfam database (Table S3). Among the annotated unigenes from T3 and T4, 32072 unigenes (98.78\%) were annotated to Nr, $22773(70.14 \%)$ to Swiss-prot, 18525 (57.05\%) to GO, 12913 (39.77\%) to KEGG, 10603 (32.66\%) to COG and 22845 (70.36\%) to Pfam database (Table S3).

DEGs related flower opening were screened from comparisons of MA-T1 vs. MA-T2 and AE-T1 vs. AE-T2 with FDR (False Discovery Rate) $<0.1$ and FC (Fold Change) $\geq 2$. DEGs related flower closing were filtered from comparisons of MA-T3 vs. MA-T4 and AE-T3 vs. AE-T4 with FDR $<0.1$ and FC $\geq 1.5$. In the comparison of MA-T1 vs. MA-T2, a total of 5832 unigenes were differentially expressed (2986 up-regulated and 2846 down-regulated), in which 4581 unigenes were annotated against the six public databases (Nr, Swissprot, GO, KEGG, COG and Pfam) (Fig. 2; Table S4 and Table S5). In the comparison of AE-T1 vs. AE-T2, a total of 4711 DEGs (3718 
annotated) were identified, in which 2910 were up-regulated and 1801 were down-regulated (Fig. 2, Table S4 and Table S5). In the comparison of MA-T3 vs. MA-T4, a total of 975 unigenes (828 annotated) were differentially expressed (493 up-regulated and 482 down-regulated) (Fig. 2, Table S4 and Table S5). In the comparison of AE-T3 vs. AE-T4, a total of 2272 DEGs (1948 annotated) were screened, in which 831 were up-regulated and 1441 were down-regulated (Fig. 2, Table S4 and Table S5).

Table 1

The sampled time and sampled blooming types for transcriptome sequencing

\begin{tabular}{|lllll|}
\hline $\begin{array}{l}\text { The time points within a } \\
\text { day }\end{array}$ & $\begin{array}{l}\text { The time of MA } \\
\text { starting opening (T1, } \\
9: 30)\end{array}$ & $\begin{array}{l}\text { The time of AE } \\
\text { starting opening (T2, } \\
\mathbf{1 4}: 30)\end{array}$ & $\begin{array}{l}\text { The time of MA } \\
\text { starting closing (T3, } \\
\mathbf{1 8}\end{array}$ & $\begin{array}{l}\text { The time of AE } \\
\text { starting closing (T4, } \\
\text { 20:00) }\end{array}$ \\
\hline $\begin{array}{l}\text { morning-opening and } \\
\text { afternoon-closing plant } \\
\text { (MA) }\end{array}$ & MA-T1 & MA-T2 & MA-T3 & MA-T4 \\
\hline $\begin{array}{l}\text { afternoon-opening and } \\
\text { evening-closing plant (AE) }\end{array}$ & AE-T 1 & AE-T2 & AE-T3 & AE-T4 \\
\hline
\end{tabular}

\section{KEGG pathway and GO enrichment analysis of DEGs}

KEGG pathway and GO enrichment analysis of unigenes differentially expressed at different flowering stages provided an overview into the gene function or pathways associated with flower opening and closing. For flower opening process, "amino acid transmembrane transport", "water transport", "circadian rhythm" and "fructose and mannose metabolism" were significantly enriched in DEGs of the both comparisons of MA-T1 vs. MA-T2 (SO stage vs. FO stage) and AE-T1 vs. AE-T2 (B stage vs. SO stage) (Fig. 3). "Cell wall biogenesis", "xyloglucan metabolic process", "xyloglucan: xyloglucosyl transferase activity", "plant hormone signal transduction" and "starch and sucrose metabolism" were specifically enriched in up-regulated gene sets of AE-T1 vs. AE-T2 (B stage vs. SO stage) (Fig. 3). In the comparison of MA-T1 vs. MA-T2 (SO stage vs. FO stage), "autophagy" and "acid phosphatase activity" were specifically enriched in up-regulated gene sets (Fig. 3).

As for flower closing process, "cell wall organization", "circadian rhythm" and "starch and metabolism" were dramatically overrepresented in DEGs of both comparisons of MA-T3 vs. MA-T4 (SC stage vs. FC stage) and AE-T3 vs. AE-T4 (FO stage vs. SC stage) (Fig. 4). Of these terms or pathways, cell wall organization term was over-represented in up-regulated gene sets of MA-T3 vs. MA-T4 (SC stage vs. FC stage), and in down-regulated gene sets of AE-T3 vs. AE-T4 (FO stage vs. SC stage) (Fig. 4). Circadian rhythm pathway was enriched in down-regulated gene sets of the both comparisons (MA-T3 vs. MA-T4 and AE-T3 vs. AE-T4). Terms (pathways) specifically enriched in up-regulated gene sets of AE-T3 vs. AE-T4 included carbon-carbon lyase activity, hydrolase activity (acting on glycosyl bonds) and plant hormone signal transduction (Fig. 4). Terms (pathways) specifically enriched in down-regulated gene sets of AE-T3 vs. AE-T4 included glucan metabolic process, pectin catabolic process and pectinesterase activity (Fig. 4). Protein processing in endoplasmic reticulum and ribosome pathway were specifically enriched in down-regulated gene sets and up-regulated gene sets of MA-T3 vs. MA-T4, respectively (Fig. 4).

\section{Transcriptional regulatory mechanism of differential flower opening and closing times between different plants}

The purpose of this study was to use RNA-seq on the same time-course transcriptomes of two plants with different within-day flowering course to identify key candidate genes regulating flower opening and closing times. Genes that show different change trends of transcript abundance between MA and $\mathrm{AE}$ on the same time-course but different flowering course are valuable for understanding the regulatory mechanisms of flower opening and closing times. The above enrichment analysis and previous reports suggest that flower opening and closing (senescing) would be involved in light input, circadian clock, hormonal regulation, water transport, osmoregulation, cell wall organization and developmental programmed cell death (dPCD). The expression levels and transcript change trends in abundance of these related genes were displayed with heat maps and the detailed data was shown in Table S8.

\section{Light input and circadian clock}

In this study, flowers of a single hybrid plant (or a species) start opening and closing at exactly the same time of different days, even in constant darkness (unpublished data). These facts suggested that flower opening and closing times of I. dichotoma, l. domestica and their hybrids were controlled by circadian clock. Furthermore, the flower opening and closing times of $I$. dichotoma and $I$. 
domestica changed with different light-dark cycles (unpublished data), suggesting the involvement of light input in the regulation of flower opening and closing times. The light- and clock- regulation of flower opening and closing times also have been reported in many other species, such as Eustoma grandiflorum and Portulaca umbraticola [8, 9].

In flower opening process (from T1 to T2), two genes associated with circadian clock and light signaling showed different expression profiles between MA and AE. The putative core clock gene EARLY FLOWERING 4-LIKE 4 (ELF4-LIKE 4) was evidently down-regulated in the comparison of MA-T1 vs. MA-T2 but up-regulated in the comparison of AE-T1 vs. AE-T2 (Fig. 5B). Another gene encodes PHYTOCHROME-INTERACTING FACTOR4-like (PIF4-like), whose homologous gene in Arabidopsis thaliana functions as a signaling hub downstream of external signals (light and temperature) and intrinsic signals from the circadian clock [25]. Its transcript abundance significantly increased in MA-T1 vs. MA-T2 (SO stage vs. FO stage) but declined in AE-T1 vs. AE-T2 (B stage vs. SO stage) (Fig. 5A). In flower closing process (from T3 to T4), PIF4-like also presented a different change trend of transcript abundance between MA and AE. A transcript encoding PIF4-like was normally regulated in MA-T3 vs. MA-T4 (SC stage vs. FC stage) but up-regulated in AET3 vs. AE-T4 (FO stage vs. SC stage) (Fig. 5C).

\section{Hormones}

Plant hormones can orchestrate multiple cellular processes that impact physiology and growth. The regulation of hormones on flower opening has been reported in Iris species [26], as well as flower closing caused by senescence [27].

In flower opening process (from T1 to T2), six gene transcripts associated with auxin synthesis and signaling were detected to present opposite change trends of abundance between MA and AE (Fig. 6A). Two gene transcripts (YUCCA2-like and YUCCA10) involved in auxin biosynthesis [28] were down-regulated in MA-T1 vs. MA-T2 (SO stage vs. FO stage) but up-regulated in AE-T1 vs. AE-T2 (B stage vs. SO stage) (Fig. 6A). A transcript encoding auxin-responsive protein SMUX AUXIN UP RNA 64-like (SAUR64-like), and a transcript encoding auxin-induced protein 22D were also detected to present up-regulation in MA-T1 vs. MA-T2 but down-regulation in AE-T1 vs. AE-T2 (Fig. 6A). Five transcripts encoding auxin efflux carrier component 1 and auxin efflux carrier component 7-like did not show significant differential expression in MA-T1 vs. MA-T2, but were up-regulated in AE-T1 vs. AE-T2 (Fig. 6A). However, the putative auxin efflux carrier component 5 and PIN-LIKES 7-like were down-regulated in the both comparisons of MA-T1 vs. MA-T2 and AE-T1 vs. AET2 (Fig. 6A). In Arabidopsis thaliana, the PINFORMED (PIN) efflux carriers function as auxin transporters. Among the PIN family proteins, PIN1 and PIN7 are localized at the plasma membrane and function as auxin efflux carriers [29, 30]. However, PIN5 and PINLIKES (PILS) proteins are localized in the endoplasmic reticulum (ER) and facilitate intracellular auxin transport between the cytosol and ER to regulate auxin homeostasis $[29,31]$. The putative auxin efflux carrier components and PILS detected in this study belong to PIN family and the function of their proteins may correspond to the PIN proteins of $A$. thaliana.

Five transcripts involved in gibberellin synthesis and signaling were detected to perform different change trends of abundance between MA and TE in flower opening process (from T1 and T2) (Fig. 6A). Among them, two transcripts encoding ent-kaurenoic acid oxidase (KAO), which are involved in biosynthesis of gibberellin [32] were up-regulated in AE-T1 vs. AE-T2 but normally regulated in MA-T1 vs. MA-T2 (Fig. 6A). The other two transcripts encoding the DELLA protein GAl-like were down-regulated in AE-T1 vs. AE-T2 but did not show significant expressional changes in MA-T1 vs. MA-T2 (Fig. 6A). DELLA proteins function as negative regulators in gibberellin signaling [33].

Only one gene transcript related to cytokinin was detected to have different change trends of abundance between MA and AE in flower opening process (from T1 to T2). The transcript encodes cytokinin dehydrogenase 5-like, a protein associated with degradation of cytokinins [34]. It was up-regulated in MA-T1 vs. MA-T2 but normally regulated in AE-T1 and AE-T2 (Fig. 6A).

Jasmonate (JA)-related gene transcripts almost showed the same change trends of abundance between MA and AE in flower opening processes (from T1 to T2). A gene transcript encoding an enzyme involved in biosynthesis of jasmonates (allene oxide cyclase, AOC) [35], and a transcript encoding ninja-family protein involved in jasmonate signaling [35], were down-regulated in the both comparisons of MA-T1 vs. MA-T2 and AE-T1 vs. AE-T2 (Fig. 6A). Two gene transcripts encoding cytochrome P450 enzyme CYP94B3 were upregulated in the both comparisons of MA-T1 vs. MA-T2 and AE-T1 vs. AE-T2 (Fig. 6A). The CYP94B3 is reported to cause the hydroxylation of JA-lle which may switch off JA/JA-lle signaling $[35,36]$.

In flower closing (senescing) process (from T3 to T4), only three auxin-related gene transcripts showed different change trends of abundance between MA and AE. Two gene transcripts encoding auxin-responsive proteins (SAUR64-like and SAUR68-like) were normally regulated in MA-T3 vs. MA-T4, but were down-regulated in AE-T3 vs. AE-T4 (Fig. 6B). A gene transcript encoding auxin efflux

Page 5/26 
carrier component 5-like (functioning as intracellular auxin transporter) was down-regulated in MA-T3 vs. MA-T4 but up-regulated in $A E-T 3$ vs. AE-T4 (Fig. 6B). Almost all gene transcripts associated with gibberellin and cytokinin presented the same change trends of abundance between $M A$ and $A E$ in flower senescing process and there were no cytokinin-related DEGs in the two comparisons of MAT3 vs. MA-T4 and AE-T3 vs. AE-T4 (Fig. 6B). Two gene transcripts encoding CYP94B3 involved in jasmonate metabolism were downregulated in AE-T3 vs. AE-T4, but did not show significant transcript changes in MA-T3 vs. MA-T4 (Fig. 6B).

\section{Water transport and osmoregulation}

Flower opening is generally due to cell expansion driven by intracellular turgor pressure $[1,18]$. The increase of intracellular turgor pressure is caused by water flowing into the cell and most water transports across the plasma membrane are mediated by aquaporins [18]. Aquaporins are mostly localized to the plasma membrane or vacuolar membrane, which are respectively called plasma membrane intrinsic proteins (PIPs) and tonoplast intrinsic proteins (TIPs) [37]. In flower opening process (from T1 to T2), two gene transcripts encoding PIP2-5 and PIP2-2 were detected to perform down-regulation in MA-T1 vs. MA-T2 but up-regulation in AE-T1 vs. AE-T2 (Fig. 7A). The gene transcript encoding TIP2-3 showed the same change trend of abundance in the two comparisons (Fig. 7A).

Water flows through aquaporins along the water potential gradient. Hence, water transports can be indirectly regulated by changing osmotic pressure and the change of osmotic pressure can be achieved by changing the cytosolic concentration of osmo-active molecules. The three major categories of osmo-active molecules modulated in osmoregulation are ions, sugars and amino acids [37]. Osmoregulation via ions predominantly occurs through $\mathrm{K}^{+}$and its counterions [37]. Gene transcripts involved in transports of $\mathrm{K}^{+}$and $\mathrm{H}^{+}$did not show different change trends of abundance in the two comparisons of MA-T1 vs. MA-T2 and AE-T1 vs. AE-T2 (Fig. 7A). A gene transcript encoding sugar carrier protein $C$ relating to glucose transmembrane import was down-regulated in MA-T1 vs. MA-T2 but up-regulated in AE-T1 vs. AE-T2 (Fig. 7A). As for amino acid, proline is particularly targeted in osmoregulation [37]. Its concentration is regulated at the levels of biosynthesis, transport and catabolism. Two gene transcripts encoding pyrroline-5carboxylate synthase (P5CS) were down-regulated in MA-T1 vs. MA-T2 but up-regulated in AE-T1 vs. AE-T2 (Fig. 7A). The rate-limiting step of proline biosynthesis is catalyzed by P5CS [38].

In flower closing process (from T3 to T4), most gene transcripts involved in water transmembrane transport, transport of $\mathrm{K}^{+}$and $\mathrm{H}^{+}$ and sugar transport were down-regulated in AE-T3 vs. AE-T4, but did not show significant differential expression in MA-T3 vs. MA-T4 (Fig. 7B).

\section{Cell wall-related activity}

Cell walls are dynamic structures with rigidity and extensibility. The extensibility of cell walls allows cell expansion which is driven by a strong intracellular turgor pressure [39]. Several gene transcripts whose proteins involve regulating the extensibility of cell walls were detected to show opposite change trends of abundance in the two comparisons of MA-T1 vs. MA-T2 and AE-T1 vs. AE-T2. In flower opening process (from T1 to T2), two gene transcripts encoding fasciclin-like arabinogalactan proteins (AGP2 and AGP12) and a gene transcript encoding xyloglucan endotransglucosylase/hydrolase (XTH) were down-regulated in MA-T1 vs. MA-T2 but up-regulated in AE-T1 vs. AE-T2 (Fig. 8A). AGPs are structural proteins in the cell wall which can regulate wall expansion[40]. XTHs can modulate the interactions between cellulose microfibrils (CMFs) and hemicellulose xyloglucans (XyGs) to strengthen or loosen cell wall [39, 41, 42].

Cell wall degradation is part of petal senescence [19]. In flower closing (senescing) process (from T3 to T4), a large number of gene transcripts relating cell wall activity were detected to present different change trends of abundance between MA and AE. Thirteen gene transcripts were normally regulated in MA-T3 vs. MA-T4 but down-regulated in AE-T3 vs. AE-T4 (Fig. 8B). These gene transcripts included four transcripts encoding AGPs (AGP12 and AGP14), a transcript encoding expansin-A16-like, three transcripts encoding cellulose synthase $A$ (CESA, mediating the biosynthesis of CMFs) [43], a transcript encoding pectin methylesterase (PME, decreasing the crosslinking between pectins and calcium ions to soften of the wall) $[44,45]$ and four transcripts encoding pectate lyases (PLs, depolymerizing pectic chains so as to loosen cell walls) [46]. Flower senescence was also associated with an increase in the transcript abundance of putative genes encoding $\beta$-galactosidase (BGAL), $\beta$-glucosidase (BGLU) and polygalacturonase (PG) which are putatively involved in cell wall degradation $[19,47]$. In flower senescing process (from T3 to T4), a gene transcript encoding BGAL and two transcripts encoding BGLU were up-regulated in AE-T3 vs. AE-T4 but did not show significant differential expression in MA-T3 and MA-T4. Two gene transcripts encoding PG-like were down-regulated in MA-T3 vs. MA-T4 but up-regulated in AE-T3 vs. AE-T4 (Fig. 8B). In constant, three gene transcripts encoding polygalacturonase inhibitor-like (PGI-like) were up-regulated in MA-T3 vs. MA-T4 but down-regulated in AE-T3 vs. AE-T4 (Fig. 8B). 


\section{Execution of senescence}

Flower senescence is a highly developmentally regulated process which is induced by programmed cell death (PCD) of petal cells.

Caspases involved in cell death signal transduction of animal cells are cysteine endo-proteases [48]. In flower senescing process (from T3 to T4), several gene transcripts whose proteins are cysteine proteases were detected to perform different change trends of abundance between MA and AE. Two of them encoding senescence-specific cysteine protease SAG39-like were down-regulated in MAT3 vs. MA-T4 but up-regulated in AE-T3 vs. AE-T4 (Fig. 9). Another transcript encoding metacaspase-9 did not show significant differential expression in MA-T3 vs. MA-T4 but was up-regulated in AE-T3 vs. AE-T4 (Fig. 9). Flower senescence is accompanied by a series of metabolic processes, such as sugar metabolism, protein degradation, remobilization and transport of mineral ions, fatty acid degradation and phospholipid degradation. Several gene transcripts associated with these processes were detected to show upregulation in AE-T3 vs. AE-T4 but show little transcript changes in MA-T3 vs. MA-T4 (Fig. 9). These gene transcripts included a transcript encoding sucrose synthase 4, three transcripts encoding proteases (cysteine protease and aspartic protease) relating to protein degradation [19], a transcript encoding isocitrate lyase-like (ICL-like) which functions in the fatty acid degradation [19, 49], two transcripts encoding phospholipase $\mathrm{D}$ alpha 1 and non-specific phospholipase $\mathrm{C} 2$ which function in the phospholipid degradation [19], and a transcript encoding purple acid phosphatase 23 which can remobilize phosphates [19]. Syntaxin are required for the fusion of transport vesicles to target membranes [50]. The two gene transcripts that encode syntaxins were down-regulated in AE-T3 vs. AET4 but did not show significant differential expression in MA-T3 vs. MA-T4 (Fig. 9).

\section{RNA-seq validation and expression analysis using qRT-PCR}

To verify the reliability of the transcriptome sequencing data, eleven DEGs were selected randomly to perform qRT-PCR on the same sample as those in transcriptome sequencing. The results showed that the expression levels of the selected genes were basically consistent with the RNA-seq results (Fig. 10), which confirmed the validity of the RNA-seq data and the following analysis.

\section{Discussion}

The plant circadian clock is involved in the regulation of numerous processes including flower opening and closing. The circadian clock can modulate the periods and phases of biological processes in response to abiotic and biotic signals [14]. The different flower opening and closing times among different species or individuals could be resulted from the different phases of transcriptional events regulating flower opening and closing. These transcriptional events have been reported to be associated with light signaling, circadian clock, hormonal regulation, water transport, osmoregulation, cell wall organization and developmental programmed cell death (dPCD) $[1,2,11,12,19,23]$, in line with the results of GO and KEGG enrichment analysis (Fig. 3 and 4). In this study, two individuals with divergent flower opening and closing times selected from the F2 hybrids of I. dichotoma and I. domestica were used to RNAsequencing to screen the key genes regulating flower opening and closing. For this purpose, we screened genes related to above processes which presented different change trends of transcript abundance between different individuals on the same time-course but different flowering course.

\section{The regulation of flower opening}

Stationary plants perceive the constantly changing environmental conditions and modulate physiological processes to optimize performance and reproductive success. Light and temperature are the most important environmental signals that regulate plant growth and development. Transcription factor PIF4 controls downstream gene expression in response to external signals such as light and temperature, as well as intrinsic signals from the circadian clock, sugars and hormones [25, 51, 52]. Firstly, binding to red lightactivated Pfr form of PHYB induced phosphorylation and subsequent degradation of PIF4 protein (Fig. 11A) [53]. The activity of PIF4 was also controlled by temperature through transcriptional and post-transcriptional mechanisms (Fig. 11 A) [54-56]. Blue lightsensing CRY1 photoreceptor restricts the transcriptional activity of PIF4 at low temperature [57] and inhibits PIF4-induced expression of auxin biosynthesis genes at high temperature [58]. Furthermore, the expression of PIF4 presents circadian rhythm which is mediated in part by the evening complex (EC) (Fig. 11A), an integral part of the circadian clock that consists of ELF3, ELF4 and LUX ARRHYTHMO (LUX) [59]. After integrating these external and intrinsic signals, PIF4 modulates multiple downstream developmental processes such as stomatal opening and hypocotyl elongation $[51,60]$. In this study, transcripts of PIF4-like detected in hybrids of $I$. dichotoma and I. domestica was up-regulated in MA-T1 vs. MA-T2 (SO stage vs. FO stage) but down-regulated in AE-T1 vs. AE-T2 (B stage vs. SO stage), suggesting that flower opening would be negatively regulated by PIF4-like. However, the putative clock

Page 7/26 
component ELF4-LIKE 4 showed down-regulation in MA-T1 vs. MA-T2 versus up-regulation in AE-T1 vs. AE-T2, which was opposite to the result of PIF4-like (Fig. 11A). These results indicate that ELF4-LIKE 4 would positively regulate flower opening, and there would be transcriptionally negative interaction between PIF4-like and ELF4-LIKE 4, which need to be verified.

In flower opening process (from T1 to T2), several putative genes involved in auxin synthesis (YUC10 and YUC2-like), auxin efflux transport (auxin efflux carrier component 1,7) and signaling (SAUR64-like and auxin-induced protein 22D) were up-regulated in AE-T1 vs. AE-T2 (B stage vs. SO stage) but down-regulated or normally regulated in MA-T1 vs. MA-T2 (SO stage vs. FO stage) (Fig. 11B). These results indicate that auxin would positively regulate flower opening through the coordination of auxin synthesis, auxin efflux and signaling. Endogenous auxins also have been demonstrated to be the regulators of flower opening in Iris $\times$ hollandica through applying exogenous hormones and inhibitors of hormones [26]. Transcriptome of flowers at different flowering stages reveals that auxin also triggers the flower opening of Nymphaea nouchali via the coordination of synthesis, homeostasis, efflux and signaling of auxin [12].

Flower opening has been reported to be triggered by petal cell expansion and auxin promotes cell expansion via increasing wall extensibility $[18,39,61]$. Auxin would trigger the transcription of auxin-responsive factor SAUR64-like whose protein can activate plasma membrane $\mathrm{H}^{+}$-ATPases, which pump $\mathrm{H}^{+}$into the wall, causing apoplast acidincation (Fig. 11B) [62, 63]. On the one hand, auxin-induced acidic $\mathrm{pH}$ of cell wall activates potassium channels which transport potassium ions $\left(\mathrm{K}^{+}\right)$to the cytosol [64], leading to the increase of osmotic pressure of cytoplasm. The increase of osmotic pressure stimulates water $\left(\mathrm{H}_{2} \mathrm{O}\right)$ uptake, which generates turgor pressure, forcing the cell wall to extend (Fig. 11B). Aquaporins play a major role in water transfer across the plasma membrane. In flower opening process, PIP2-5 and PIP2-2 encoding aquaporins were down-regulated in MA-T1 vs. MA-T2 but up-regulated in AE-T1 vs. AE-T2, suggesting the positive regulation of PIPs in flower opening (Fig. 11B). The involvement of aquaporins in flower opening has been reported in many species, such as Rosa cv. 'Samantha', Tulipa and Dianthus caryophyllus [65-67].

In addition to ions $\left(\mathrm{K}^{+}\right)$, sucrose transported by sugar carrier protein $\mathrm{C}$ and proline synthesized by P5CS-like also play a key role in osmoregulation and stimulating water uptake [18]. Sugar carrier protein $C$ and P5CS-like presented down-regulation in MA-T1 vs. MAT2 but up-regulation in AE-T1 vs. AE-T2, indicating they would positively regulate flower opening (Fig. 11B). In Capparis spinosa, the highest proline and sugar concentrations occurred at flower opening [68].

On the other hand, auxin-induced wall acidification promotes the expression of genes which induce wall loosening such as XTH [39, 69]. In flower opening process, the putative AGPS (AGP2 and AGP12) and XTH improving cell wall extensibility were down-regulated in MA-T1 vs. MA-T2 but up-regulated in AE-T1 vs. AE-T2, suggesting the increase of petal cell-wall extensibility during the rapid flower opening process (from B stage to SO stage). In Ipomoea nil, the petal elongation and the flower opening rate (controlled by the darkperiod length) were closely correlated with the expression patterns of XTHs in petals [70].

Previous studies and results of this study demonstrates that hypocotyl growth, stomatal opening and flower opening are all involved in the activation of $\mathrm{H}^{+}$-ATPaes by SAUR proteins $[62,71,72]$. Moreover, hypocotyl growth and stomatal opening have been demonstrated to be regulated by PIF4 [51,60], results of this study also suggest that flower opening would be modulated by PIF4-like. In Arabidopsis thaliana, PIF4 combining with BZR1 (BRASSINAZOLE-RESISTANT1) promotes the expression of auxin-related genes, thus promotes hypocotyl growth [51, 73]. However, stomatal opening was inhibited by PIF4 because pif4 mutants exhibit wider open stomata [60]. The mechanisms by which PIF4 regulates stomatal opening and the interactions between PIF4 and auxin in stomatal opening have not been clarified. Transcriptome profiles of this study suggest that flower opening would be negatively regulated by PIF4-like. The functions of PIF4-like and other candidate genes regulating flower opening still need to be verified, and the regulatory mechanism by which PIF4-like regulates flower opening also need to be investigated.

\section{The regulation of flower closing}

In flower closing (senescing) process (from T3 to T4), PIF4-like was up-regulated in AE-T3 vs. AE-T4 but did not show significant differential expression in MA-T3 vs. MA-T4 (Fig. 5C), indicating that PIF4-like would positively modulate the start of flower senescence. SAUR64-like and SAUR68-like encoding auxin-responsive factors, and CYP94B3 whose protein causing the hydroxylation of JA-lle which turns off JA signaling [35, 36], were down-regulated in AE-T3 vs. AE-T4 (FO stage vs. SO stage), but showed few transcript changes in MA-T3 vs. MA-T4 (Fig. 6B). Genes related to gibberellin and cytokinin did not show different change trends of abundance between the two comparisons (Fig. 6B). These results indicate that endogenous auxin and jasmonate may influence the flower senescence, while gibberellin and cytokinin would have no effect on flower senescence. Flowers of Iris are ethylene-independent 
[74]. Although the influence of other hormones on ethylene-independent Iris flowers has been analyzed through applying exogenous and hormone inhibitors [27], there are as yet no convincing data showing that any hormone controls the onset of their senescence.

In flower closing process, genes encoding aquaporin, potassium channels, $\mathrm{Na}^{+} / \mathrm{H}^{+}$antiporter and sugar carrier protein $\mathrm{C}$ were all down-regulated in AE-T3 vs. AE-T4 but normally regulated in MA-T3 and MA-T4 (Fig. 7B). From FO stage (fully opening stage) to SC stage (starting senescing stage), the decreased expression of these genes related to water transport and osmoregulation suggest the decrease of cellular turgor pressure would be associated with the start of flower closure. It has been reported that closure of singleopening flowers is resulted from turgor loss due to senescence [1]. Considering that the transcription of aquaporins is modulated by the circadian clock [37], it is likely that circadian clock regulation of flower opening and closing occurs partially via aquaporins.

Most of genes related to wall organization showed different expression patterns between MA and AE in flower closing (senescing) process. Among these genes, genes encoding AGPs, EXPA16, PME, PLs (improving the wall loosening) and genes encoding CESAs (synthesizing cellulose microfibrils) were down-regulated in AE-T3 vs. AE-T4 (form FO stage to SC stage), but did not express differentially significantly in MA-T3 and MA-T4 (SC stage vs. FC stage) (Fig. 8B). These results indicate that the decrease of cell wall extensibility and cellulose microfibril levels would be associated with the start of flower senescence. Three genes promoting cell wall degradation, $B G A L, B G L U$ (encoding enzymes related to cell wall degradation) and PG-like, were significantly up-regulated in AE-T3 vs. AE-T4, but were normally regulated or down-regulated in MA-T3 vs. MA-T4 (Fig. 8B), implying that cell wall degradation would occur at the start of flower senescence. In several other species, the transcript abundance of $\beta$-galactosidase (Ipomea; Sandersonia; Hemerocallis) [47, 75, 76], $\beta$-glucosidase (Ipomoea) [75] and PG (Hemerocallis) [47] also increase during senescence.

The genes which were up-regulated in AE-T3 vs. AE-T4 but down-regulated or normally regulated in MA-T3 vs. MA-T4 encode enzymes or proteins involved in protein degradation independent of proteasome, sucrose synthesis, fatty acid degradation, phospholipid degradation and remobilization of mineral ions. These results demonstrate that the increasing expression levels of these genes are correlated with the start of flower senescence. The proteins encoded by these genes function in the execution of senescence.

\section{Allelic variation alters flower opening and closing times by influencing input pathways of clock}

The differences in daily rhythms of gene expression result in different flower opening and closing times between MA and AE. However, how natural genetic variation modulates temporal patterns of expression during the day is poorly understood. The allelic variation causing variable flower opening and closing times may occur at the input pathways of external signals, the core oscillator generating the rhythms or output pathways producing rhythmic behavior. Allelic variation of the core oscillator components would disrupt rhythmic expression of core circadian clock and output genes [77], while natural variation occurring at input pathways could adjust the activity of clock-regulated processes while avoiding the pleiotropic effects with severe disruptions of circadian system. For example, the delayed phase of circadian rhythm in cultivated tomato than its wild ancestor is associated with allelic variation in the tomato homolog of EMPFINDLICHER IM DUNKELROTEN LICHT1 (EID1) which is involved in phytochrome signaling in Arabidopsis [78]. In 77 Arabidopsis accessions, natural variation in the timing of GI (GIGANTEA) expression is explained by natural alleles that alter light sensitivity of GI [79]. In both I. dichotoma and I. domestica, flower opening and closing, as well as the expression of clock and output genes keep robust circadian rhythms even in continuous darkness (unpublished data). Moreover, upstream clock-related genes ELF4-LIKE 4 and PIF4-like are adjusted to perform different expression patterns between MA and AE. These results suggest that allelic variation would alter flower opening and closing times by influencing input pathways of external signals, rather than circadian rhythm. The components underlying the genetic variation can be further investigated with QTL mapping.

\section{Conclusions}

Two individuals with significantly divergent flower opening and closing times were selected from the F2 hybrids of $I$. dichotoma and $I$. domestica for transcriptome sequencing to explore the regulatory mechanisms of flower opening and closing (senescing) times. In the putative regulatory network of flower opening time, the upstream clock-related gene PIF4-like integrate external signals (light and temperature) and intrinsic signals from the circadian clock (the transcriptional inhibition of ELF4-LIKE 4) to negatively modulate flower opening time. In the midstream, auxin would positively regulate flower opening through the coordination of auxin synthesis (YUC10 and YUC2-like), auxin efflux (auxin efflux carrier component 1,7) and auxin signaling (SAUR64-like) since the expression of these genes increased from bud stage to starting opening stage (AE-T1 to AE-T2) but decreased form starting opening stage to full opening stage (MA-T1 to MA-T2). In the downstream, auxin-responsive factor SAUR64-like triggers cell wall acidification through 
activating $\mathrm{H}^{+}$-ATPase. On the one hand, cell wall acidification activates inward $\mathrm{K}^{+}$channels to increase cellular osmotic pressure, which stimulates water uptake and augments turgor pressure, forcing the cell wall to extend. In this process, PIP2-2 and PIP2-5 encoding aquaporins were up-regulated in AE-T1 vs. AE-T2 but down-regulated in MA-T1 vs. MA-T2, suggesting the positive regulation of PIPS in flower opening. On the other hand, cell wall acidification promotes the expression of genes triggering wall loosening, for example, XTH were down-regulated in MA-T1 vs. MA-T2 but up-regulated in AE-T1 vs. AE-T2. In addition to $\mathrm{K}^{+}$, sugar transported by sugar carrier protein $\mathrm{C}$ and proline synthesized by P5CS-like are also osmo-active molecules regulating osmotic pressure. Sugar carrier protein $C$ and $P 5 C S$-like were up-regulated in AE-T1 vs. AE-T2 but down-regulated in MA-T1 vs. MA-T2, indicating their positive regulation in flower opening.

PIF4-like would positively regulate the start of flower closing. Flower senescence is a kind of developmental programmed cell death (dPCD) and is accompanied with turgor loss of petal cell. In flower senescing process (from FO stage to SC stage), the activities of cell wall synthesis and loosening, aquaporins, transmembrane transport of $\mathrm{K}^{+}$and $\mathrm{H}^{+}$, sugar transmembrane transport were decreased. However, the activities of cell wall degradation, protein degradation independent of proteasome, sucrose synthesis, fatty acid degradation, phospholipid degradation and remobilization of mineral ions were increased at the start of flower senescence.

\section{Materials And Methods}

\section{Plant materials and sample collection}

Iris domestica (L.) Goldblatt \& Mabb. and Iris dichotoma Pall., are two wild Iris species which are widely distributed in China. Wild plants of $I$. domestica and I. dichotoma used in this study were collected in the Dayang Mountain National Forestry Park $\left(40^{\circ} 16^{\prime} 60^{\prime \prime} \mathrm{N}\right.$, $116^{\circ} 27^{\prime} 58^{\prime \prime} \mathrm{E}$ ) and no permission is required to collect these plants. We identified these collected plants according to the voucher specimens (BJFC00054908 for I. domestica and BJFC00058371 for I. dichotoma) preserved in the Museum of Beijing Forestry University. A plant of I. dichotoma was crossed with a plant of $I$. domestica to obtain the F1 hybrids. Then a plant of the F1 hybrids was artificially pollinated with pollen of itself to obtain the F2 hybrids. The F2 hybrids varied widely in flower opening time (varied from 7:00 to 14:45) and closing time (varied from 19:00 to 23:00) [24]. All plant materials were cultivated in the open field of China National Engineering Research Center for Floriculture $\left(40^{\circ} 17^{\prime} \mathrm{N}, 116^{\circ} 39^{\prime} \mathrm{E}\right)$ and were given the same soil condition, moisture condition, temperature and light conditions (including light intensity and photoperiod).

Two individuals with significantly different flower opening and closing times were selected from the F2 hybrids of I. dichotoma and $I$. domestica for transcriptome sequencing. Their flowering processes were categorized into 5 stages according to our previous study [24]: flower bud stage (B stage), the stage when flower petals start opening (SO stage), the stage when petals are fully opened (FO stage), the stage when petals start closing (SC stage), the stage when petals are fully closed and form a tube (FC stage) (Fig. 1). Flower opening time and closing time mentioned in this study refer to the time of flower starting opening (the time of SO stage) and the time of flower starting closing (the time of SC stage), respectively. One of the two selected individuals started opening at 9:30 (SO stage) and started to close at 18:15 (SC stage), which was named as MA (morning-opening and afternoon-closing plant). Another individual started opening at 14:30 (SO stage) and started closing at 20:00 (SC stage), which was named as AE (afternoon-opening and evening-closing plant). On a sunny day of July 2019, blooming flowers (or flower buds which would open on the day) of each selected plant were harvested at four time points over a single day: 9:30 (T1), 14:30 (T2), 18:15 (T3) and 20:00 (T4) which are flower opening and closing times of the two plants (Table 1). All collected tissues were immediately frozen in liquid nitrogen and stored at $-80^{\circ} \mathrm{C}$ for RNA extraction. Three biological replicates were performed for each flower sample for a total of 24 samples in RNA-seq analysis.

\section{RNA extraction, library construction and RNA-seq}

Total RNA of each sample was isolated using the Quick RNA Isolation Kit (Huayueyang, Beijing, China). The purity, integrity and concentration of RNA were assessed with agarose gel electrophoresis, Nano-Photometer spectrophotometer (IMPLEN, CA, USA) and the Qubit RNA Assay Kit in Qubit 2.0 Fluorometer (Life Technologies, CA, USA). Eight libraries were generated using NEBNext ${ }^{B} U$ Itra $^{\text {TM }}$ RNA Library Prep Kit for Illumina ${ }^{\circledR}$ (NEB, USA) following manufacturer's recommendations. The library quality was assessed on the Agilent Bioanalyzer 2100 system. The cDNA libraries were sequenced on an Illumina Hiseq 2000 platform.

\section{De novo assembly and functional annotation}


Clean reads were obtained by removing reads containing adapter, reads containing unknown base " $\mathrm{N}$ " (where the " $\mathrm{N}$ " ratio was more than $10 \%$ ) and low quality reads from raw data. Transcriptome de novo assembly based on clean reads was accomplished using Trinity V2.5.1 [80] with min_kmer_cov set to 2 by default and all other parameters set default. Trinity first broke sequencing reads into shorter fragments. Then these fragments were extended into longer contigs. Then contigs were clustered based on overlaps between them to obtain component. Finally, transcription sequences called unigenes were identified using De Bruijn graphs of each component and sequencing read information. These unigenes were annotated using the BLASTx alignment (E-value $\left.\leq 10^{-5}\right)$ to following databases: NCBI non-redundant (Nr) protein database, Swiss-Prot, Gene Ontology (GO), Kyoto Encyclopedia of Genes and Genomes (KEGG) and Clusters of Orthologous Groups (COG) database. Subsequently, the unigenes were annotated to Protein family (Pfam) database using HMMER software (E-value $\left.\leq 10^{-10}\right)$.

\section{Expression annotation}

The expression levels of unigenes were estimated by RSEM [81], and FPKM (Fragments Per Kilobase of transcript per Million mapped reads) was used to represent the expression abundance. Differential expression analysis of two groups was performed using the DESeq R package (1.10.1). The $P$-values were adjusted using the Benjamini and Hochberg's approach for controlling the false discovery rate. Genes with an adjusted $P$-value (False Discovery Rate, FDR) $<0.01$ and a fold change $\geq 2$ were assigned as differentially expressed genes (DEGs) for transcriptomes at T1 and T2 (flower opening process). Genes with a FDR $<0.01$ and a fold change $\geq 1.5$ were assigned as DEGs for transcriptomes at T3 and T4 (flower closing process).

\section{GO and KEGG pathway enrichment analysis for differentially expressed unigenes}

Gene Ontology (GO) enrichment analysis of DEGs screened above was implemented by the topGO R packages based KolmogorovSmirnov test (corrected $P$-value $<0.05$ ). The statistical enrichment of DEGs in KEGG pathways were analyzed using KOBAS software [82]. The enrichment significance of each enriched pathway was tested using a two-tailed Fisher's Exact Test (corrected $P$-value $<0.05$ ). Enrichment Factor was used to represent the enrichment degree of a GO term or a KEGG pathway. It is calculated as the ratio of the proportion of DEGs annotated to a term (or a pathway) in all DEGs to the proportion of unigenes annotated to that term (or that pathway) in all unigenes annotated to GO database (KEGG pathway database).

\section{RNA-seq validation and expression analysis using qRT-PCR}

The results of transcriptome sequencing were validated using real-time quantitative PCR (RT-qPCR) on eleven randomly selectedgenes differentially expressed in flower opening and/or closing processes. The RNA used in gene validation was identical with the RNA used in transcriptome sequencing (Table 1). Primer sequences were designed with Primer Premier 5 (Table S9). cDNA systhesis and RT-qPCR were performed using PrimeScript ${ }^{\text {TM }}$ RT reagent Kit (with gDNA Eraser) and TB Green ${ }^{\circledR}$ Premix Ex Taq ${ }^{\text {TM }}$ II (SYBR Green) (Takara BioTech (Beijing) Co., Ltd., China). Real-time quantitative PCR in this study were carried out on a CFX96 Real-Time PCR Detection System (Bio-Rad). Three independent biological replicates for each sample and three technical replicates of each biological replicate were performed in all experiments. GAPDH (glyceraldehyde-3-phosphate dehydrogenase) was selected as an internal control [83]. The relative expression level was calculated according to the $2^{-\Delta \Delta C t}$ algorithm.

\section{Abbreviations}

AE: afternoon-opening and evening-closing plant; MA: morning-opening and afternoon-closing plant; B stage: The flower bud stage; SO stage: The stage when flower petals start opening; FO stage: The stage when petals are fully opened; SC stage: The stage when petals start closing; FC stage: The stage when petals are fully closed and form a tube.

\section{Declarations}

\section{Ethics approval and consent to participate}

Not applicable

\section{Consent for publication}

Not applicable 
The raw reads have been submitted to NCBI SRA (Sequence Read Archive, http://www.ncbi.nlm.nih.gov/sra/) under the accession number PRJNA729120, and NCBI TSA (Transcriptome Shotgun Assembly, https://www.ncbi.nlm.nih.gov/nucleotide/) under the accession number GJFB00000000.

\section{Competing interests}

The authors declare that they have no competing interests.

\section{Funding}

This work was supported by the National Natural Science Foundation of China (No. 31971706) and the Fundamental Research Funds for the Central Universities (No. 2019YC08). The funders did not have any role in the design of the study, collection, analysis, or interpretation of data or the writing of the manuscripts.

\section{Authors' contributions}

All authors contributed to the study conception and design. Material preparation, data collection and analysis were performed by RL and YG. The first draft of the manuscript was written by RL and all authors commented on previous versions of the manuscript. All authors read and approved the final manuscript.

\section{Acknowledgements}

We thank Lili Ruan for helping us to establish the hybrid population. We also thank Guobin Kou for the daily management for our plant materials.

\section{References}

1. van Doorn WG, Van Meeteren U. Flower opening and closure: a review. Journal of experimental botany. 2003; 54(389):18011812.

2. van Doorn WG, Kamdee C. Flower opening and closure: an update. Journal of experimental botany. 2014; 65(20):5749-5757.

3. Matsumoto T, Yasumoto AA, Nitta K, Yahara T, Tachida H. Difference in flowering time as an isolating barrier. Journal of theoretical biology. 2013; 317:161-167.

4. Liu R, Gao Y, Fan Z, Wang X, Xiao J, Zhang Q. Within-day temporal isolation of two species of Iris (Iridaceae) sharing the same pollinator. Biol J Linn Soc. 2020; 130(3):447-457.

5. Julia C, Dingkuhn M. Variation in time of day of anthesis in rice in different climatic environments. Eur J Agron. 2012; 43:166174.

6. Bheemanahalli R, Sathishraj R, Manoharan M, Sumanth HN, Muthurajan R, Ishimaru T, Krishna JS. Is early morning flowering an effective trait to minimize heat stress damage during flowering in rice? Field Crops Res. 2017; 203:238-242.

7. Horibe T, Yamada K. Petals of cut rose flower show diurnal rhythmic growth. J Jpn Soc Hortic Sci. 2014; 83(4):302-307.

8. Bai J, Kawabata S. Regulation of diurnal rhythms of flower opening and closure by light cycles, wavelength, and intensity in Eustoma grandiflorum. J Jpn Soc Hortic Sci. 2015; 84(2):148-155.

9. Maguvu TE, Higuchi Y, Shibata M. Effect of different photoperiods on flower opening time in Portulaca umbraticola. J Jpn Soc Hortic Sci. 2017; 87(1):124-131.

10. Yon F, Joo Y, Cortés Llorca L, Rothe E, Baldwin IT, Kim SG. Silencing Nicotiana attenuata LHY and ZTL alters circadian rhythms in flowers. The New phytologist. 2016; 209(3):1058-1066.

11. Trivellini A, Cocetta G, Hunter DA, Vernieri P, Ferrante A. Spatial and temporal transcriptome changes occurring during flower opening and senescence of the ephemeral hibiscus flower, Hibiscus rosa-sinensis. Journal of experimental botany. 2016; 67(20):5919-5931.

12. Ke M, Gao Z, Chen J, Qiu Y, Zhang L, Chen X. Auxin controls circadian flower opening and closure in the waterlily. BMC plant biology. 2018; 18(1):143. 
13. McWatters HG, Devlin PF. Timing in plants--a rhythmic arrangement. FEBS letters. 2011; 585(10):1474-1484.

14. Webb AAR, Seki M, Satake A, Caldana C. Continuous dynamic adjustment of the plant circadian oscillator. Nature communications. 2019; 10(1):550.

15. Nohales MA, Kay SA. Molecular mechanisms at the core of the plant circadian oscillator. Nature structural \& molecular biology. 2016; 23(12):1061-1069.

16. Oakenfull RJ, Davis SJ. Shining a light on the Arabidopsis circadian clock. Plant, cell \& environment. $2017 ; 40(11): 2571-2585$.

17. Sanchez SE, Rugnone ML, Kay SA. Light perception: a matter of time. Molecular plant. 2020; 13(3):363-385.

18. Beauzamy L, Nakayama N, Boudaoud A. Flowers under pressure: ins and outs of turgor regulation in development. Ann Bot. 2014; 114(7):1517-1533.

19. van Doorn WG, Woltering EJ. Physiology and molecular biology of petal senescence. Journal of experimental botany. 2008; 59(3):453-480.

20. Shahri W, Tahir I. Flower senescence: some molecular aspects. Planta. 2014; 239(2):277-297.

21. Xu X, Gookin T, Jiang C-Z, Reid M. Genes associated with opening and senescence of Mirabilis jalapa flowers. Journal of experimental botany. 2007; 58(8):2193-2201.

22. Yamada T, Ichimura K, Kanekatsu M, van Doorn WG. Gene expression in opening and senescing petals of morning glory (Ipomoea nil) flowers. Plant cell reports. 2007; 26(6):823-835.

23. van Doorn WG, Balk PA, van Houwelingen AM, Hoeberichts FA, Hall RD, Vorst O, van der Schoot C, van Wordragen MF. Gene expression during anthesis and senescence in Iris flowers. Plant molecular biology. 2003; 53(6):845-863.

24. Liu R, Gao Y, Ruan L, Fan Z, Li C. Variation of flower opening and closing times in hybrids of evening flowering species Iris dichotoma and daytime flowering species Iris domestica. Plant Breeding. 2018; 137(6):920-927.

25. Balcerowicz M. PHYTOCHROME-INTERACTING FACTORS at the interface of light and temperature signalling. Physiologia plantarum. 2020; 169(3):347-356.

26. van Doorn WG, Dole I, Celikel FG, Harkema H. Opening of Iris flowers is regulated by endogenous auxins. J Plant Physiol. 2013; 170(2):161-164.

27. van Doorn WG, Çelikel FG, Pak C, Harkema H. Delay of iris flower senescence by cytokinins and jasmonates. Physiologia plantarum. 2013; 148(1):105-120.

28. Fukui K, Hayashi KI. Manipulation and sensing of auxin metabolism, transport and signaling. Plant \& cell physiology. 2018; 59(8):1500-1510.

29. Adamowski M, Friml J. PIN-dependent auxin transport: action, regulation, and evolution. The Plant cell. $2015 ; 27(1): 20-32$.

30. Naramoto S. Polar transport in plants mediated by membrane transporters: focus on mechanisms of polar auxin transport. Current opinion in plant biology. 2017; 40:8-14.

31. Barbez E, Kubeš M, Rolčík J, Béziat C, Pěnčík A, Wang B, Rosquete MR, Zhu J, Dobrev PI, Lee Y et al. A novel putative auxin carrier family regulates intracellular auxin homeostasis in plants. Nature. 2012; 485(7396):119-122.

32. Salazar-Cerezo S, Martínez-Montiel N, García-Sánchez J, Pérez YTR, Martínez-Contreras RD. Gibberellin biosynthesis and metabolism: A convergent route for plants, fungi and bacteria. Microbiological research. 2018; 208:85-98.

33. Ito T, Okada K, Fukazawa J, Takahashi Y. DELLA-dependent and -independent gibberellin signaling. Plant signaling \& behavior. 2018; 13(3):1-3.

34. Schmülling T, Werner T, Riefler M, Krupková E, Bartrina y Manns I. Structure and function of cytokinin oxidase/dehydrogenase genes of maize, rice, Arabidopsis and other species. Journal of plant research. 2003; 116(3):241-252.

35. Wasternack C, Hause B. Jasmonates: biosynthesis, perception, signal transduction and action in plant stress response, growth and development. An update to the 2007 review in Annals of Botany. Ann Bot. 2013; 111(6):1021-1058.

36. Heitz T, Widemann E, Lugan R, Miesch L, Ullmann P, Désaubry L, Holder E, Grausem B, Kandel S, Miesch M et al. Cytochromes P450 CYP94C1 and CYP94B3 catalyze two successive oxidation steps of plant hormone Jasmonoyl-isoleucine for catabolic turnover. The Journal of biological chemistry. 2012; 287(9):6296-6306.

37. Chaumont F, Tyerman SD. Aquaporins: highly regulated channels controlling plant water relations. Plant physiology. 2014; 164(4):1600-1618. 
38. Hu CA, Delauney AJ, Verma DP. A bifunctional enzyme (delta 1-pyrroline-5-carboxylate synthetase) catalyzes the first two steps in proline biosynthesis in plants. Proc Natl Acad Sci U S A. 1992; 89(19):9354-9358.

39. Majda M, Robert S. The role of auxin in cell wall expansion. International journal of molecular sciences. 2018; 19(4).

40. Cassab G, I.. Plant cell wall proteins. Annu Rev Plant Physiol Plant Mol Biol 1998; 49(1):281-309.

41. Fry SC, Smith RC, Renwick KF, Martin DJ, Hodge SK, Matthews KJ. Xyloglucan endotransglycosylase, a new wall-loosening enzyme activity from plants. The Biochemical journal. 1992; 282 (Pt 3)(Pt 3):821-828.

42. Vissenberg K, Fry SC, Pauly M, Höfte H, Verbelen JP. XTH acts at the microfibril-matrix interface during cell elongation. Journal of experimental botany. 2005; 56(412):673-683.

43. Somerville C. Cellulose synthesis in higher plants. Annual review of cell and developmental biology. 2006; 22:53-78.

44. Virk SS, Cleland RE. The role of wall calcium in the extension of cell walls of soybean hypocotyls. Planta. 1990; 182(4):559-564.

45. Hocq L, Pelloux J, Lefebvre V. Connecting homogalacturonan-type pectin remodeling to acid growth. Trends in plant science. 2017; 22(1):20-29.

46. Marín-Rodríguez MC, Orchard J, Seymour GB. Pectate lyases, cell wall degradation and fruit softening. Journal of experimental botany. 2002; 53(377):2115-2119.

47. Panavas T, Reid PD, Rubinstein B. Programmed cell death of daylily petals: activities of wall-based enzymes and effects of heat shock. Plant Physiol Bioch. 1998; 36(5):379-388.

48. Hail N, Jr., Carter BZ, Konopleva M, Andreeff M. Apoptosis effector mechanisms: a requiem performed in different keys. Apoptosis: an international journal on programmed cell death. 2006; 11(6):889-904.

49. Chen ZH, Walker RP, Acheson RM, Tecsi LI, Astrid W, Lea PJ, Leegood RCJP. Are isocitrate lyase and phosphoenolpyruvate carboxykinase involved in gluconeogenesis during senescence of barley leaves and cucumber cotyledons? Plant \& cell physiology. 2000; 41:960-967.

50. Sanderfoot AA, Pilgrim M, Adam L, Raikhel NV. Disruption of individual members of Arabidopsis syntaxin gene families indicates each has essential functions. The Plant cell. 2001; 13(3):659-666.

51. de Lucas M, Prat S. PIFs get BRright: PHYTOCHROME INTERACTING FACTORs as integrators of light and hormonal signals. The New phytologist. 2014; 202(4):1126-1141.

52. Shor E, Paik I, Kangisser S, Green R, Huq E. PHYTOCHROME INTERACTING FACTORS mediate metabolic control of the circadian system in Arabidopsis. The New phytologist. 2017; 215(1):217-228.

53. Leivar P, Monte E, Cohn MM, Quail PH. Phytochrome signaling in green Arabidopsis seedlings: impact assessment of a mutually negative phyB-PIF feedback loop. Molecular plant. 2012; 5(3):734-749.

54. Koini MA, Alvey L, Allen T, Tilley CA, Harberd NP, Whitelam GC, Franklin KA. High temperature-mediated adaptations in plant architecture require the bHLH transcription factor PIF4. Current biology: CB. 2009; 19(5):408-413.

55. Foreman J, Johansson H, Hornitschek P, Josse EM, Fankhauser C, Halliday KJ. Light receptor action is critical for maintaining plant biomass at warm ambient temperatures. The Plant journal: for cell and molecular biology. 2011; 65(3):441-452.

56. Yamashino T, Nomoto Y, Lorrain S, Miyachi M, Ito S, Nakamichi N, Fankhauser C, Mizuno T. Verification at the protein level of the PIF4-mediated external coincidence model for the temperature-adaptive photoperiodic control of plant growth in Arabidopsis thaliana. Plant signaling \& behavior. 2013; 8(3):e23390.

57. Zhou Y, Xun Q, Zhang D, Lv M, Ou Y, Li J. TCP Transcription Factors Associate with PHYTOCHROME INTERACTING FACTOR 4 and CRYPTOCHROME 1 to Regulate Thermomorphogenesis in Arabidopsis thaliana. iScience. 2019; 15:600-610.

58. Ma D, Li X, Guo Y, Chu J, Fang S, Yan C, Noel JP, Liu H. Cryptochrome 1 interacts with PIF4 to regulate high temperature-mediated hypocotyl elongation in response to blue light. Proc Natl Acad Sci U S A. 2016; 113(1):224-229.

59. Nusinow DA, Helfer A, Hamilton EE, King JJ, Imaizumi T, Schultz TF, Farré EM, Kay SA. The ELF4-ELF3-LUX complex links the circadian clock to diurnal control of hypocotyl growth. Nature. 2011; 475(7356):398-402.

60. Wang FF, Lian HL, Kang CY, Yang HQ. Phytochrome B is involved in mediating red light-induced stomatal opening in Arabidopsis thaliana. Molecular plant. 2010; 3(1):246-259.

61. Rayle DL, Cleland RE. The acid growth theory of auxin-induced cell elongation is alive and well. Plant physiology. 1992; 99(4):1271-1274. 
62. Takahashi K, Hayashi K, Kinoshita T. Auxin activates the plasma membrane H+-ATPase by phosphorylation during hypocotyl elongation in Arabidopsis. Plant physiology. 2012; 159(2):632-641.

63. Spartz AK, Ren H, Park MY, Grandt KN, Lee SH, Murphy AS, Sussman MR, Overvoorde PJ, Gray WM. SAUR inhibition of PP2C-D phosphatases activates plasma membrane H+-ATPases to promote cell expansion in Arabidopsis. The Plant cell. 2014; 26(5):2129-2142.

64. Thiel G, Weise R. Auxin augments conductance of K+ inward rectifier in maize coleoptile protoplasts. Planta. 1999; 208(1):38-45.

65. Ma N, Xue J, Li Y, Liu X, Dai F, Jia W, Luo Y, Gao J. Rh-PIP2;1, a rose aquaporin gene, is involved in ethylene-regulated petal expansion. Plant physiology. 2008; 148(2):894-907.

66. Azad AK, Sawa Y, Ishikawa T, Shibata H. Phosphorylation of plasma membrane aquaporin regulates temperature-dependent opening of tulip petals. Plant \& cell physiology. 2004; 45(5):608-617.

67. Morita S, Sugiyama S, Tateishi A, Satoh S. Identification and characterization of plasma membrane intrinsic protein (PIP) aquaporin genes in petals of opening carnation flowers. Horticulture Journal. 2017; 86(1):78-86.

68. Rhizopoulou S, loannidi E, Alexandredes N, Argiropouios A. A study on functional and structural traits of the nocturnal flowers of Capparis spinosa L. J Arid Environ. 2006; 66(4):635-647.

69. Van Sandt VS, Suslov D, Verbelen JP, Vissenberg K. Xyloglucan endotransglucosylase activity loosens a plant cell wall. Ann Bot. 2007; 100(7):1467-1473.

70. Shinozaki Y, Tanaka R, Ono H, Ogiwara I, Kanekatsu M, van Doorn WG, Yamada T. Length of the dark period affects flower opening and the expression of circadian-clock associated genes as well as xyloglucan endotransglucosylase/hydrolase genes in petals of morning glory (Ipomoea nil). Plant cell reports. 2014; 33(7):1121-1131.

71. Wong JH, Klejchová M, Snipes SA, Nagpal P, Bak G, Wang B, Dunlap S, Park MY, Kunkel EN, Trinidad B et al. SAUR proteins and PP2C.D phosphatases regulate H+-ATPases and K+ channels to control stomatal movements. Plant physiology. 2021; 185(1):256-273.

72. Hager A. Role of the plasma membrane H+-ATPase in auxin-induced elongation growth: historical and new aspects. Journal of plant research. 2003; 116(6):483-505.

73. Oh E, Zhu JY, Wang ZY. Interaction between BZR1 and PIF4 integrates brassinosteroid and environmental responses. Nature cell biology. 2012; 14(8):802-809.

74. Çelikel FG, van Doorn WG. Endogenous ethylene does not regulate opening of unstressed Iris flowers but strongly inhibits it in water-stressed flowers. J Plant Physiol. 2012; 169(14):1425-1429.

75. Wiemken-Gehrig V, Wiemken A, Matile P. Cell wall breakdown in wilting flowers of Ipomoea tricolor Cav. Planta. 1974; 115(4):297307.

76. O'Donoghue EM, Eason JR, Somerfield SD, Ryan DA. Galactosidases in opening, senescing and water-stressed Sandersonia aurantiaca flowers. Functional plant biology: FPB. 2005; 32(10):911-922.

77. Bendix C, Marshall CM, Harmon FG. Circadian clock genes universally control key agricultural traits. Molecular plant. 2015; 8(8):1135-1152.

78. Müller NA, Wijnen CL, Srinivasan A, Ryngajllo M, Ofner I, Lin T, Ranjan A, West D, Maloof JN, Sinha NR et al. Domestication selected for deceleration of the circadian clock in cultivated tomato. Nature genetics. 2016; 48(1):89-93.

79. de Montaigu A, Giakountis A, Rubin M, Tóth R, Cremer F, Sokolova V, Porri A, Reymond M, Weinig C, Coupland G. Natural diversity in daily rhythms of gene expression contributes to phenotypic variation. Proc Natl Acad Sci U S A. 2015; 112(3):905-910.

80. Grabherr MG, Haas BJ, Yassour M, Levin JZ, Thompson DA, Amit I, Adiconis X, Fan L, Raychowdhury R, Zeng Q et al. Full-length transcriptome assembly from RNA-Seq data without a reference genome. Nature biotechnology. 2011; 29(7):644-652.

81. Li B, Dewey CN. RSEM: accurate transcript quantification from RNA-Seq data with or without a reference genome. BMC bioinformatics. 2011; 12:323.

82. Xie C, Mao X, Huang J, Ding Y, Wu J, Dong S, Kong L, Gao G, Li CY, Wei L. KOBAS 2.0: a web server for annotation and identification of enriched pathways and diseases. Nucleic acids research. 2011;39(Web Server issue):W316-322.

83. Gu CS, Liu LQ, Deng YM, Zhu XD, Lu XQ, Huang SZ. Validation of reference genes for RT-qPCR normalization in Iris lactea var. chinensis leaves under different experimental conditions. Sci Hortic. 2014; 175:144-149. 


\section{Figures}

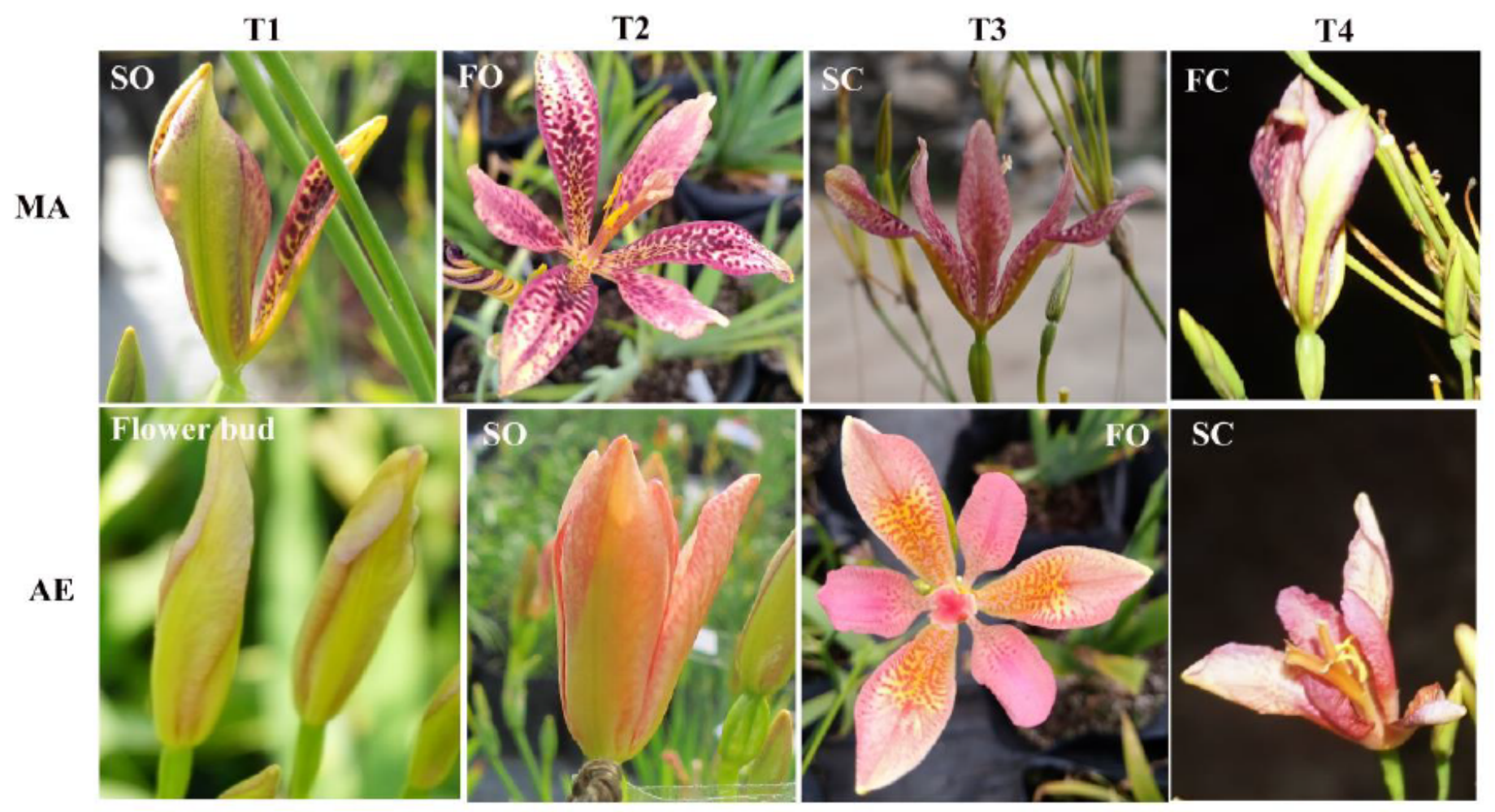

\section{Figure 1}

The flowering stages of MA (morning-opening and afternoon-closing plant) and AE (afternoon-opening and evening-closing plant) at sampled time (T1, T2, T3 and T4) of transcriptome sequencing. Their flowering processes were categorized into 5 stages according to previous study[24][24][24]: flower bud stage (B stage), the stage when flower petals start opening (SO stage), the stage when petals are fully opened (FO stage), the stage when petals starting closing (SC stage), the stage when petals are fully closed and form a tube (FC stage) 


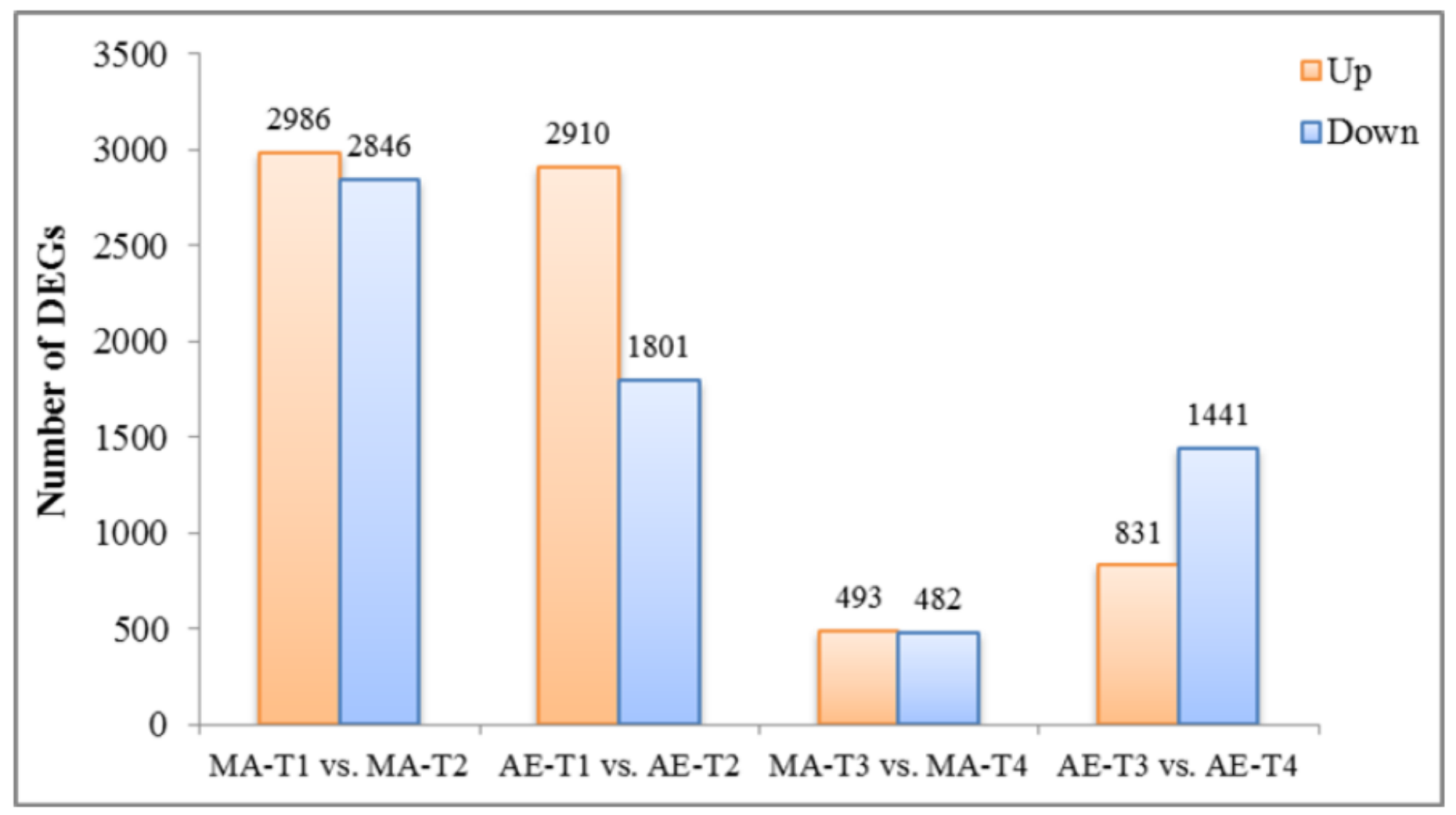

\section{Figure 2}

The number of DEGs in different comparisons, including MA-T1 vs. MA-T2, AE-T1 vs. AE-T2, MA-T3 vs. MA-T4 and AE-T3 vs. AE-T4 


\begin{tabular}{|c|c|c|c|}
\hline Category & Term & $\begin{array}{l}\text { MA-T1 vs. } \\
\text { MA-T2 }\end{array}$ & $\begin{array}{l}\text { AE-T1 vs. } \\
\text { AE-T2 }\end{array}$ \\
\hline GOTERM_BP & Amino acid transmembrane transport & & \\
\hline GOTERM_BP & Autophagy & & \\
\hline GOTERM_BP & Cell wall biogenesis & & \\
\hline GOTERM_BP & Inorganic anion transport & & \\
\hline GOTERM_BP & Polyol transport & & \\
\hline GOTERM_BP & Protein folding & & \\
\hline GOTERM_BP & water transport & & \\
\hline GOTERM_BP & xyloglucan metabolic process & & \\
\hline GOTERM_CC & Extrinsic component of membrane & & \\
\hline GOTERM_CC & Thylakoid & & \\
\hline GOTERM_MF & Acid phosphatase activity & & \\
\hline GOTERM_MF & Carbohydrate transmembrane transporter activity & & \\
\hline GOTERM_MF & Carboxylic acid transmembrane transporter activit & & \\
\hline GOTERM_MF & Carboxypeptidase activity & & \\
\hline GOTERM_MF & Glycerol channel activity & & \\
\hline GOTERM_MF & Oxidoreductase activity & & \\
\hline GOTERM_MF & Unfolded protein binding & & \\
\hline GOTERM_MF & xyloglucan:xyloglucosyl transferase activity & & \\
\hline KEGG PATH & Circadian rhythm - plant & & \\
\hline KEGG PATH & Fructose and mannose metabolism & & \\
\hline KEGG PATH & Glycerophospholipid metabolism & & \\
\hline KEGG PATH & Glycine, serine and threonine metabolism & & \\
\hline KEGG PATH & Glycolysis / Gluconeogenesis & & \\
\hline KEGG PATH & Pentose phosphate pathway & & \\
\hline KEGG PATH & Phenylpropanoid biosynthesis & & \\
\hline KEGG PATH & Carbon fixation in photosynthetic organisms & & \\
\hline KEGG PATH & Plant hormone signal transduction & & \\
\hline KEGG PATH & Plant-pathogen interaction & & \\
\hline KEGG PATH & Protein processing in endoplasmic reticulum & & \\
\hline KEGG PATH & Starch and sucrose metabolism & & \\
\hline KEGG PATH & Tyrosine metabolism & & \\
\hline
\end{tabular}

\section{Figure 3}

Gene Ontology (GO) and KEGG pathway enrichment analysis of unigenes differentially expressed by 2-fold or more in the comparisons of MA-T1 vs. MA-T2 (SO stage vs. FO stage) and AE-T1 vs. AE-T2 (B stage vs. SO stage) according to functional categories. Only terms or pathways showing a enrichment factor $\geq 1.3$, a corrected P-value $₫ 0.05$, and a count of DEGs $\geq 9$ are shown. Abbreviations are as in Fig. 1. Details of terms and pathways are listed in Table S6 and S7.Red and blue represent enriched terms in the up-regulated and down-regulated gene sets, respectively 


\begin{tabular}{|c|c|c|c|}
\hline Category & Term & MA-T4 & AE-T4 \\
\hline GOTERM_BP & Cell wall organization & & \\
\hline GOTERM_BP & Glucan metabolic process & & \\
\hline GOTERM_BP & Pectin catabolic process & & \\
\hline GOTERM_CC & Extracellular region & & \\
\hline GOTERM_MF & Carbon-carbon lyase activity & & \\
\hline GOTERM_MF & Hydrolase activity, acting on glycosyl bonds & & \\
\hline GOTERM_MF & Pectinesterase activity & & \\
\hline KEGG PATH & Pentose and glucuronate interconversions & & \\
\hline KEGG PATH & Phenylalanine metabolism & & \\
\hline KEGG PATH & Phenylpropanoid biosynthesis & & \\
\hline KEGG PATH & Plant hormone signal transduction & & \\
\hline KEGG PATH & Plant-pathogen interaction & & \\
\hline KEGG PATH & Protein processing in endoplasmic reticulum & & \\
\hline KEGG PATH & Ribosome & & \\
\hline KEGG PATH & Starch and sucrose metabolism & & \\
\hline
\end{tabular}

Figure 4

Gene Ontology (GO) and KEGG pathway enrichment analysis of unigenes differentially expressed by 1.5 -fold or more in the comparisons of MA-T3 vs. MA-T4 (SC stage vs. FC stage) and AE-T3 vs. AE-T4 (FO stage vs. SC stage) according to functional categories. Only terms or pathways showing a enrichment factor $\geq 1.3$, a corrected P-value $\varangle 0.05$, and a count of $D E G s \geq 9$ are shown. Abbreviations are as in Fig. 1. Details of terms and pathways are listed in Table S6 and S7. Red, blue and overlap red/blue represent enriched terms in the up-regulated, down-regulated and both up- and down-regulated gene sets, respectively 


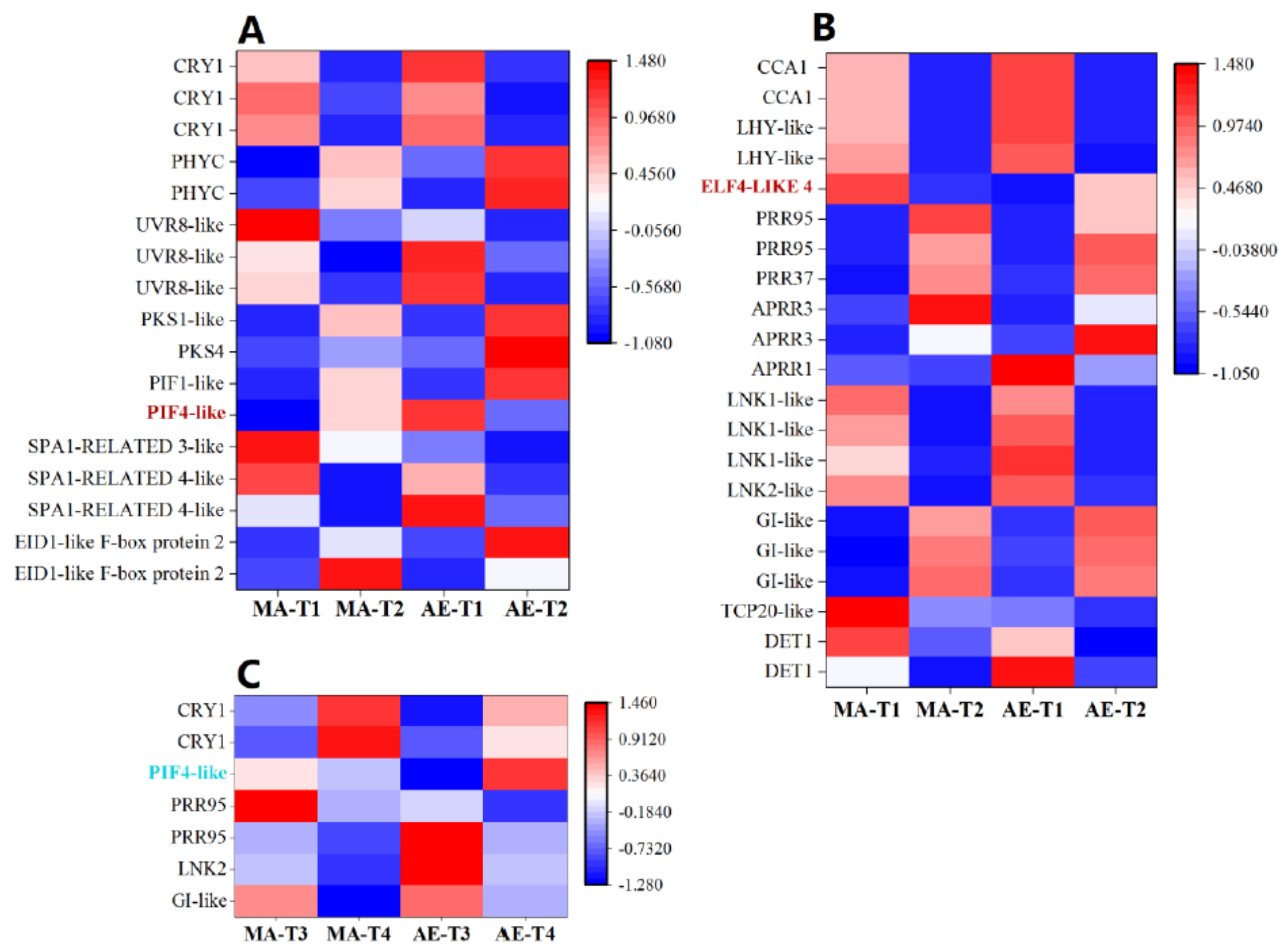

\section{Figure 5}

Expression of genes involved in light input and circadian clock in MA and AE during flower opening process (from T1 to T2) (A, B) and flower closing process (C). Data of a row were standardized with Z-score normalization. Rows and columns are not clustered. Genes with black font, red font and green font respectively represent genes with the same change trends of transcript abundance between $M A$ and $A E$, genes with opposite change trends between $M A$ and $A E$, and genes which were normally regulated in MA but differentially expressed in AE during flower opening or closing process. T1-T4 represents the four time points in Table 1. Abbreviations are as in Fig. 1. The Unigene ID, full name, expression levels and annotation of each gene are listed in Table S8 

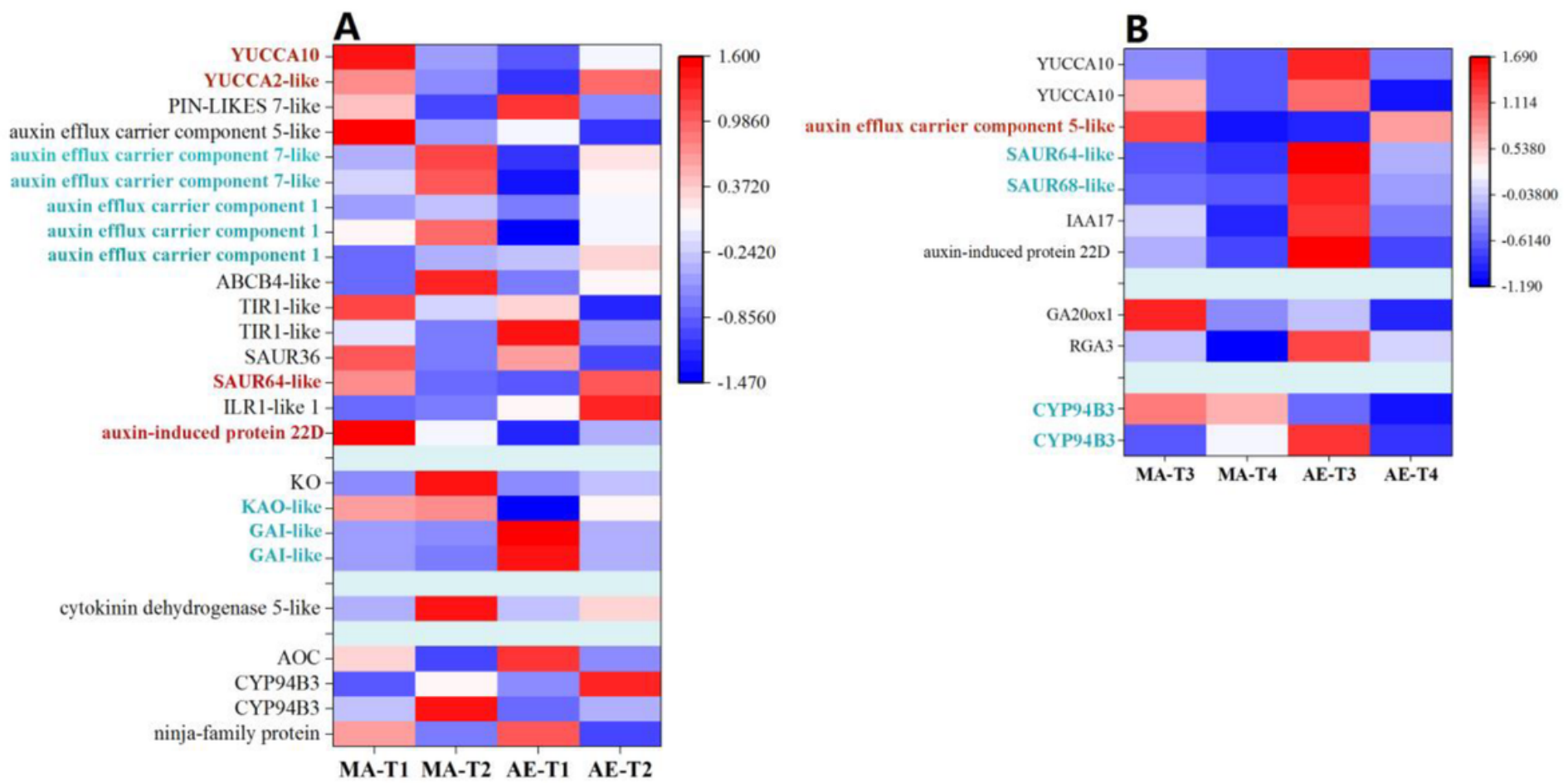

\section{Figure 6}

Expression of genes involved in hormone (auxin, gibberellin, cytokinin and jasmonate) in MA and AE during flower opening process (from T1 to T2) (a) and flower closing process (from T3 to T4) (b). Data of a row were standardized with Z-score normalization. Rows and columns are not clustered. Genes with black font, red font and green font respectively represent genes with the same change trends of transcript abundance between $M A$ and $A E$, genes with opposite change trends between $M A$ and $A E$, and genes which were normally regulated in MA but differentially expressed in AE during flower opening or closing process. Abbreviations are as in Fig. 1. The Unigene ID, full name, expression levels and annotation of each gene are listed in Table S8
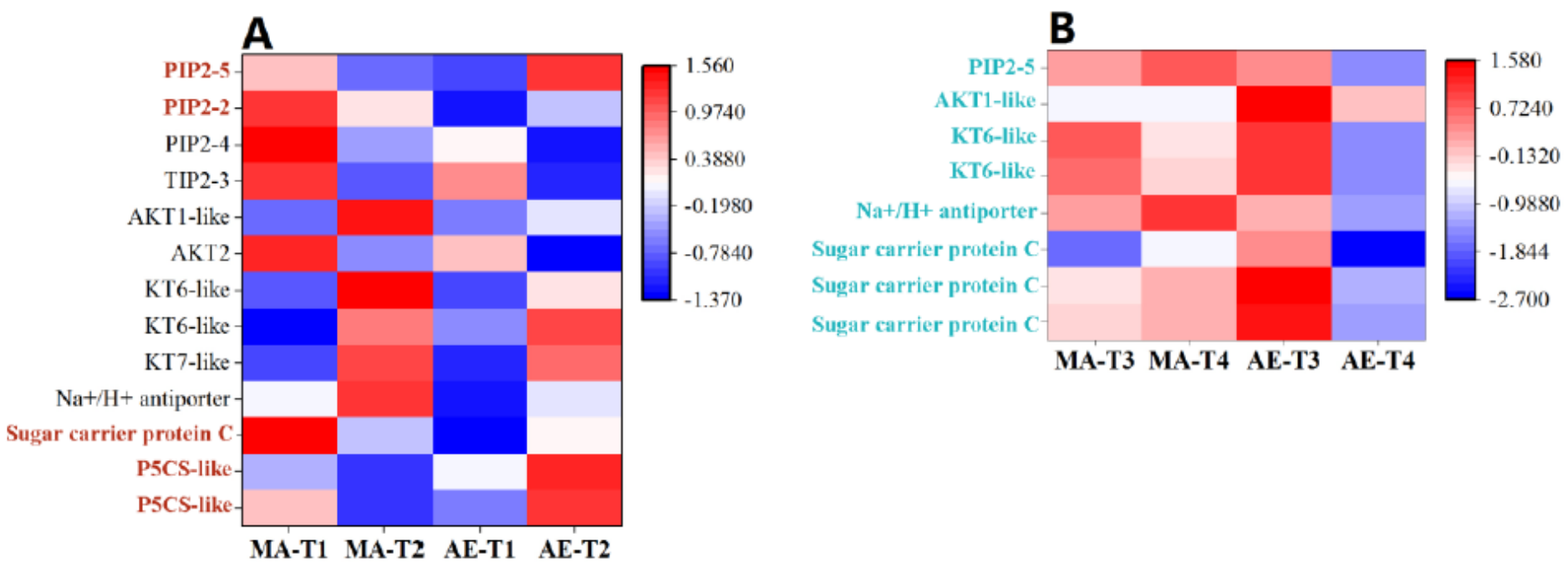

MA-T3 MA-T4 AE-T3 AE-T4

\section{Figure 7}

Expression of genes involved in water transport and osmoregulation in MA and AE during flower opening process (from T1 to T2) (a) and flower closing process (from T3 to T4) (b). Data of a row were standardized with Z-score normalization. Rows and columns are not clustered. Genes with black font, red font and green font respectively represent genes with the same change trends of transcript abundance between $M A$ and $A E$, genes with opposite change trends between $M A$ and $A E$, and genes which were normally regulated in 
MA but differentially expressed in AE during flower opening or closing process. Abbreviations are as in Fig. 1. The Unigene ID, full name, expression levels and annotation of each gene are listed in Table S8
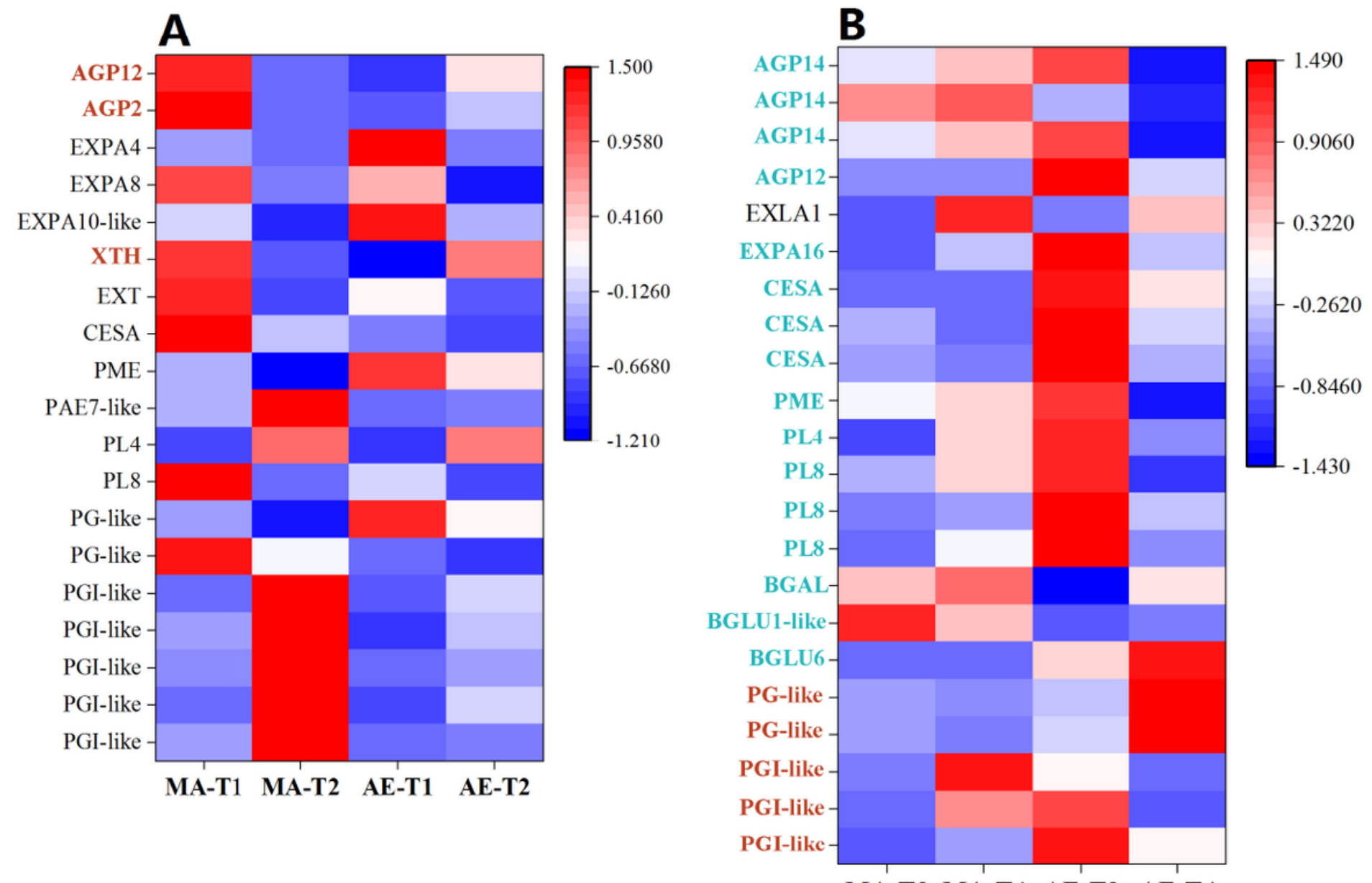

MA-T3 MA-T4 AE-T3 AE-T4

\section{Figure 8}

Expression of cell wall-related genes in MA and AE during flower opening process (from T1 to T2) (a) and flower closing process (from T3 to T4) (b). Data of a row were standardized with Z-score normalization. Rows and columns are not clustered. Genes with black font, red font and green font respectively represent genes with the same change trends of transcript abundance between $M A$ and $A E$, genes with opposite change trends between $M A$ and $A E$, and genes which were normally regulated in MA but differentially expressed in $\mathrm{AE}$ during flower opening or closing process. Abbreviations are as in Fig. 1. The Unigene ID, full name, expression levels and annotation of each gene are listed in Table S8 


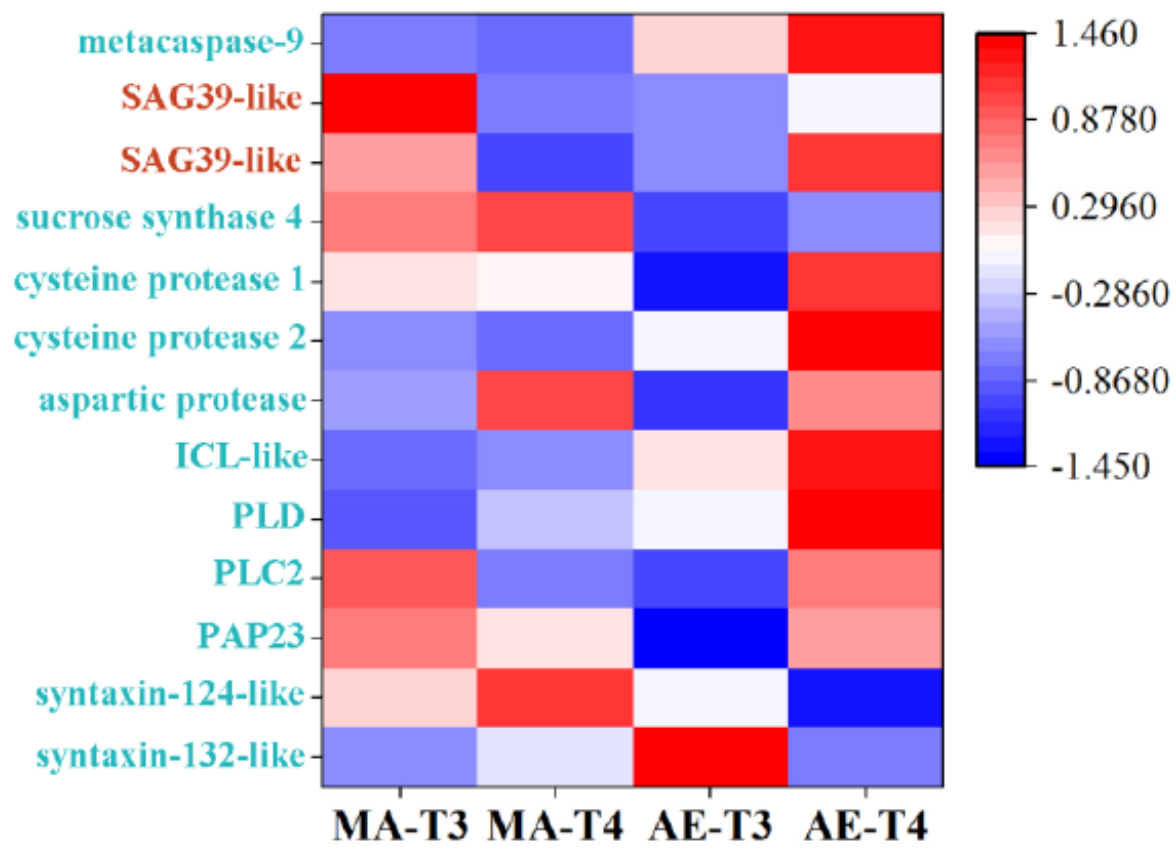

\section{Figure 9}

Expression of senescence-related genes in MA and AE during flower closing process (from T3 to T4). Data of a row were standardized with Z-score normalization. Rows and columns are not clustered. Genes with red font and green font respectively represent genes with opposite change trends between $\mathrm{MA}$ and $\mathrm{AE}$, and genes which were normally regulated in MA but differentially expressed in $\mathrm{AE}$ during flower closing process. Abbreviations are as in Fig. 1. The Unigene ID, full name, expression levels and annotation of each gene are listed in Table S8 


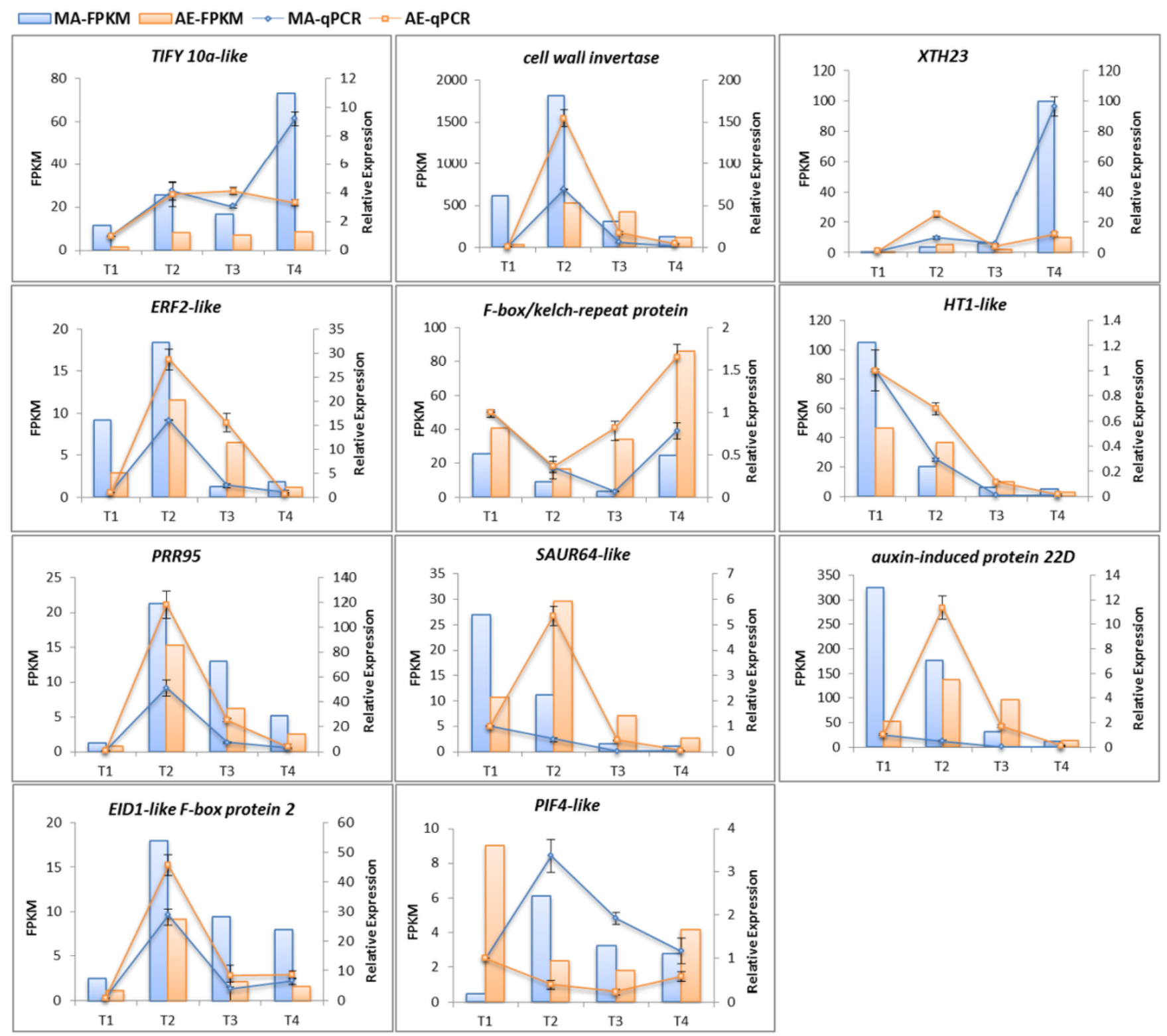

Figure 10

The expression profiles of eleven randomly selected DEGs in MA and AE. The expression patterns of TIFY 10a-like, cell wall invertase, XTH23 (xyloglucan endotransglucosylase/hydrolase protein 23), ERF2 (ETHYLEME-RESPONSIVE FACTOR 2), F-box/kelch-repeat protein, HT1-like (serine/threonine-protein kinase), PRR95 (PSEUDO-RESPONSE REGULATOR 95), SAUR64-like (SMALL AUXIN UP RNA64-like), auxin induced protein 22D, EID1-like F-box protein 2 and PIF4-like (PHYTOCHROME INTERACTING FACTOR 4-like) were determined by qRT-PCR (relative expression compared to T1) and RNA-seq analysis (FPKM values). Error bars represent the standard deviation of the means. T1-T4 represents the four time points in Table 1. MA and AE represent the morning-opening and afternoonclosing plant and afternoon-opening and evening-closing plant, respectively. 


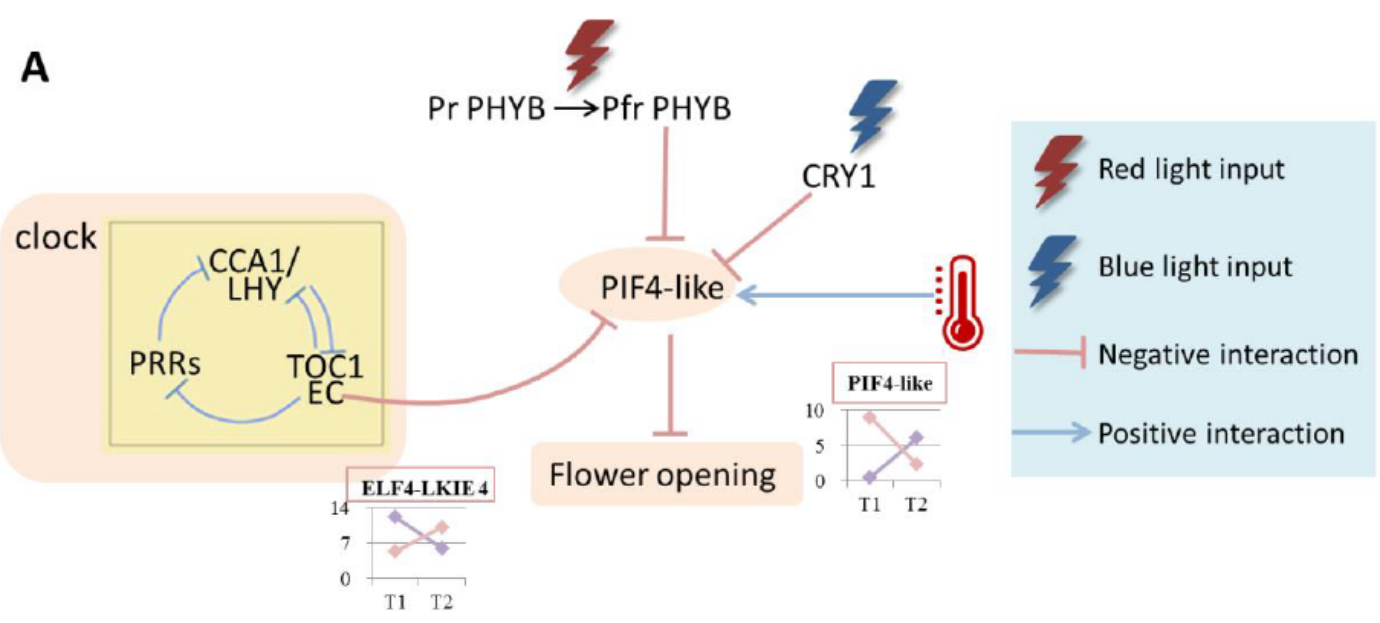

\section{B}

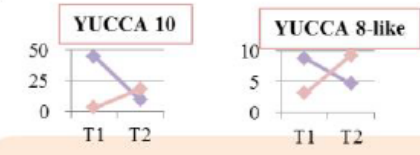

Auxin biosynthesis:

YUCCA10, YUCCA2-like

auxin efflux carrier component 1

auxin effux carrier component 1

$120 \quad$ Auxin signaling:

$60 \leftrightarrow$ SAUR64-like

T1 T2 SAUR64-like

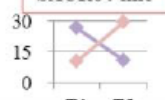

The increase of osmotically active molecules:

Sugar carrier protein C, P5CS-like

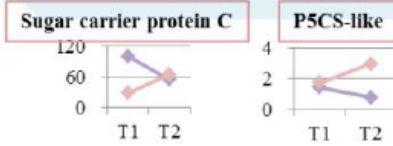

Figure 11

The putative upstream (A) and downstream (B) regulatory network of flower opening in hybrids of I. dichotoma and I. domestica. Purple lines in line charts represent expression profiles of MA (morning-opening and afternoon-closing plant), and pink lines represent expression patterns of $\mathrm{AE}$ (afternoon-opening and evening-closing plant). The ordinate is the gene expression level estimated by FPKM (Fragments Per Kilobase of transcript per Million mapped reads). T1 and T2 represent the two time points in Table 1.

\section{Supplementary Files}

This is a list of supplementary files associated with this preprint. Click to download.

- FigureS1.ThelengthdistributionoftheassembledunigenesfromT1andT2.tif

- FigureS2.ThelengthdistributionoftheassembledunigenesfromT3andT4.tif

- Supplementaryinformation.docx

- Tables1.UnigenesandPrimersusedinqRTPCR.xlsx

- Tables2.Summaryofllluminatranscriptomesequencing.xlsx

- TableS3.Summaryofllluminatranscriptomeassembly.xlsx 
- TableS4.Thefunctionalannotationsofunigenesagainstthepublicdatabases.xlsx

- TableS5.StatisticalanalysisofDEGannotationindifferentcomparisons.xlsx

- TableS6.TheexpressionlevelsandannotationsoftheDEGs.xlsx

- TableS7.TheGOenrichmentanalysisofDEGs.xlsx

- TableS8.TheKEGGpathwayenrichmentanalysisofDEGs.xlsx

- TableS9.ExpressionlevelsofselectedgenesusedforheatmapinthetwoselectedF2hybridindividualsatdifferentsampledtimepoints.xlsx 